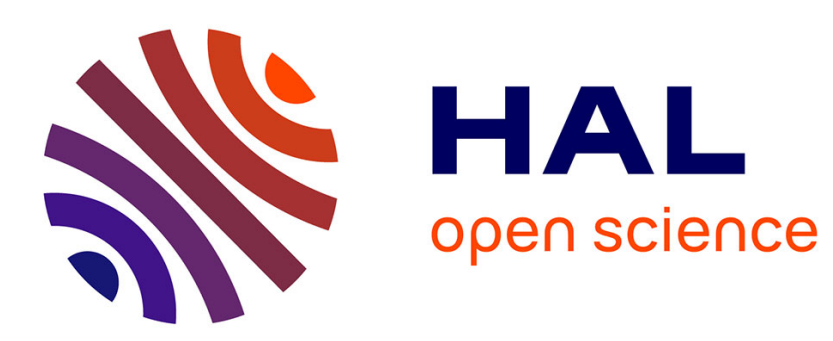

\title{
Politiques sociales, stratégies électorales et fédéralisme sous la présidence Clinton
}

\author{
François Vergniolle de Chantal, Daniel Béland
}

\section{To cite this version:}

François Vergniolle de Chantal, Daniel Béland. Politiques sociales, stratégies électorales et fédéralisme sous la présidence Clinton. Revue Française de Science Politique, 2000, 50 (6), pp.883-914. 10.3406/rfsp.2000.395523 . halshs-02508708

\section{HAL Id: halshs-02508708 \\ https://shs.hal.science/halshs-02508708}

Submitted on 15 Mar 2020

HAL is a multi-disciplinary open access archive for the deposit and dissemination of scientific research documents, whether they are published or not. The documents may come from teaching and research institutions in France or abroad, or from public or private research centers.
L'archive ouverte pluridisciplinaire HAL, est destinée au dépôt et à la diffusion de documents scientifiques de niveau recherche, publiés ou non, émanant des établissements d'enseignement et de recherche français ou étrangers, des laboratoires publics ou privés. 


\section{Politiques sociales, stratégies électorales et fédéralisme sous la présidence Clinton \\ In: Revue française de science politique, 50e année, n6, 2000. pp. 883-914.}

\section{Résumé}

L'article se présente comme une analyse des réformes sociales menées sous la présidence Clinton (1993-2001), en se concentrant sur le projet de couverture santé universelle, la réforme de l'aide sociale (1996), et les débats sur le devenir du système des retraites. Cette étude permet d'avancer un certain nombre de conclusions, tant au plan épistémologique qu'au plan poli-tique. Dans le premier cas, il est possible de relativiser le diagnostic fait par Paul Pier son sur « la nouvelle politique de l'État providence ». Bien loin de se limiter à des politiques d'évite-ment, les réformes sociales des années 1990 aux États-Unis, comportent une part substantielle de recherche de crédits électoraux. En second lieu, l'exemple des politiques sociales éclaire la nature de l'emploi du thème fédéral dans le débat public. La généralisation de l'évocation du fédéralisme est, dans le cadre de certaines limites, instrumentale et pragmatique, tout comme, d'ailleurs, le thème de la « Troisième Voie » clintonienne.

\section{Abstract}

Social policies, electoral strategies and federalism during clinton's presidency

This article presents an analysis of social policies during the Clinton presidency (1993-2001). Its focus is on health care reform, the Welfare Act of 1996 and the debates about the future of Social Security. It presents various conclusions, both epistemological and political. The developments of social policy during the 1990s in the United States make it possible to downplay Paul Pier son's diagnosis concerning «The New Politics of the Welfare State ». Blame avoi- dance strategies cannot explain the social policies of the 1990s ; electoral credit-claiming was also an important aspect. Social policies highlight the nature of federalism as an issue in public debates. The generalization of its use is, within certain limits, pragmatic and instrumental. The same can be said for Clinton's « Third Way ».

Citer ce document / Cite this document :

Vergniolle De Chantal François, Beland Daniel. Politiques sociales, stratégies électorales et fédéralisme sous la présidence Clinton. In: Revue française de science politique, 50e année, $n^{\circ} 6,2000$. pp. 883-914.

doi : $10.3406 / \mathrm{rfsp} .2000 .395523$

http://www.persee.fr/web/revues/home/prescript/article/rfsp_0035-2950_2000_num_50_6_395523

\section{(ङ) $\Theta$}




\section{POLITIQUES SOCIALES, STRATÉGIES ÉLECTORALES \\ ET FÉDÉRALISME \\ SOUS LA PRÉSIDENCE CLINTON}

\section{DANIEL BÉLAND, FRANÇOIS VERGNIOLLE DE CHANTAL}

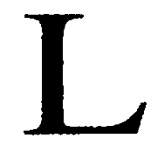

e débat sur l'avenir de la protection sociale occupe une place centrale dans la vie politique contemporaine. Aux États-Unis, ce débat se conjugue avec un effort de réforme du fédéralisme ${ }^{1}$. Stimulée par la montée de la « nouvelle droite » conservatrice, la remise en cause du Big Government - associée aux noms de Ronald Reagan et de Newt Gingrich - inscrit la question du « Nouveau Fédéralisme » au cœur de l'agenda social américain. Au cours de la présidence Clinton (1993-2001), ce qu'on peut qualifier de rhétorique antifédérale occupe toujours une place de choix aux États-Unis ${ }^{2}$. Au lendemain de la double victoire républicaine aux élections législatives de 1994, le mouvement de contestation du rôle social de l'État fédéral atteint même son apogée. La réforme de 1996 de l'aide sociale apparaît comme sa conséquence directe.

Le présent article explore les stratégies électorales poursuivies par les différents acteurs politiques concernant la réforme du système fédéral de protection sociale pendant la présidence Clinton. Pour ce faire, il convient d'analyser les trois éléments les plus fondamentaux de ce système : la santé, l'aide sociale et les retraites. Plus spécifiquement, on s'efforcera de saisir la mobilisation du thème fédéral dans le débat social américain. La compréhension de ce phénomène devrait éclairer la nature des stratégies électorales mises en œuvre par Clinton.

1. Au-delà de la question sociale elle-même, le thème du fédéralisme irrigue évidemment l'ensemble du débat public américain. Dans ce cadre, le fédéralisme représente un angle d'attaque légitime pour saisir l'évolution du débat social sous les présidences Clinton. Pour une perspective historique et comparative au sujet de l'articulation entre fédéralisme et protection sociale en Amérique du Nord, on lira l'analyse de Bruno Théret : « Du fédéralisme et de la protection sociale en Amérique et en particulier au Canada », rapport pour la convention d'étude $n^{\circ} 5$, Commissariat général du Plan, 1998. Dans son analyse, Théret souligne très justement l'écart qui existe entre les fédéralismes américain et canadien. Il y a plusieurs formes de fédéralisme, et le présent article ne porte que sur l'expérience américaine. Ses conclusions au sujet de la relation entre fédéralisme, stratégies électorales et réforme des politiques sociales ne concernent que les États-Unis.

2. L'antifédéralisme désigne une critique générale de la centralisation politique au niveau national. Par commodité, on utilisera le terme de décentralisation comme synonyme. Contrairement à ce que le sens commun pourrait laisser croire en France, l'antifédéralisme n'est donc pas synonyme d'un quelconque jacobinisme, bien au contraire. Pour une perspective historique sur la révolution américaine et les antifédéralistes de 1787, cf. Denis Lacorne, L'invention de la république. Le modèle américain, Paris, Hachette, 1991, ainsi que François Vergniolle de Chantal, «L'antifédéralisme américain. Diversité et pérennité », Commentaire, 91, automne 2000 , p. 543-548. En anglais, se reporter à Saul Cornell, The Other Founders. Anti-Federalism and the Dissenting Tradition in America, 1788-1828, Chapel Hill, University of North Carolina Press, 1999. 
Théoriquement ce travail emprunte certains concepts au néo-institutionnalisme, approche qui accorde une grande importance à l'impact des institutions sur les stratégies des acteurs et l'élaboration des politiques publiques '. Parmi les représentants du néo-institutionnalisme, Paul Pierson est très certainement 1'auteur qui propose la théorie la plus cohérente au sujet des transformations contemporaines de la protection sociale ${ }^{2}$. Selon lui, la « nouvelle politique de l'État providence » rompt franchement avec la recherche de crédits électoraux (credit-claiming) au fondement du développement des politiques sociales dans l'après-guerre ${ }^{3}$. Au lieu de rechercher les fruits électoraux associés à l'élargissement de la protection sociale, la classe politique s'efforce désormais de réduire les dépenses sociales (retrenchment) tout en diminuant les risques électoraux qui dérivent de cette opération. Pour neutraliser l'opposition des groupes de bénéficiaires qui défendent leurs « acquis sociaux », hommes politiques et hauts fonctionnaires mobilisent différentes stratégies d'évitement (blame avoidance) ${ }^{4}$. Ces stratégies visent généralement à masquer l'étendue (ou les effets) des coupes budgétaires ou à diminuer la responsabilité apparente de la classe politique au sujet des transformations intervenues.

Selon Pierson, les responsables politiques ne peuvent neutraliser qu'une partie des risques électoraux engendrés par les privatisations et les réductions budgétaires. Face notamment à l'opposition des groupes de bénéficiaires, il semble difficile, voire impossible de transformer radicalement les systèmes de protection sociale instaurés dans l'après-guerre : les programmes sociaux les plus importants survivent presque inévitablement aux attaques néolibérales. Les institutions produisent en effet des agrégations d'intérêts qui leur permettent de se reproduire malgré ces attaques ${ }^{5}$.

1. Selon Peter A. Hall et Rosemary C. R. Taylor, il existe trois variantes principales de cette approche théorique : le néo-institutionnalisme historique, le néo-institutionnalisme des choix rationnels et le néo-institutionnalisme sociologique : « La science politique et les trois néo-institutionnalismes », Revue française de science politique, 47 (3-4), juin-août 1997, p. 469-495.

2. Paul Pierson, Dismantling the Welfare State?:Reagan, Thatcher, and the Politics of Retrenchment, Oxford, Cambridge University Press, 1994 ; cf. également, Paul Pierson, « The New Politics of the Welfare State », World Politics, 48, janvier 1996, p. 143-179.

3. On peut définir la recherche de crédit comme une stratégie politique visant des gains électoraux. En vue de leur réélection, les acteurs politiques s'efforcent de cibler des groupes en leur apportant des gains économiques et sociaux par l'intermédiaire de politiques dont ils s'attribuent la responsabilité.

4. Les stratégies d'évitement servent à limiter les risques électoraux associés à des décisions qui heurtent les intérêts ou les convictions d'un ou de plusieurs groupes sociaux. En tentant de masquer leur responsabilité politique ou les conséquences de leurs décisions, les élus souhaitent prévenir la mobilisation de ces groupes et, à plus long terme, des pertes électorales significatives. Les concepts de stratégies d'évitement (blame avoidance) et de recherche de crédit (credit-claiming) ont d'abord été formulés par $\mathrm{R}$. Kent Weaver dans un article maintes fois cité : «The Politics of Blame Avoidance », Journal of Public Policy, 6 (4), 1986, p. 371398.

5. Dans ce contexte, les systèmes de protection sociale empruntent un «chemin de dépendance » (path dependency), ils ne peuvent que difficilement prendre une voie radicalement différente de celle tracée par leur histoire institutionnelle. D'abord développée par l'économiste Douglass $\mathrm{C}$. North, la notion de path dependence renvoie d'abord aux rendements croissants des institutions et aux coûts économiques qui freinent leur remplacement : Institutions, Institutional Change and Economic Performance, Cambridge, Cambridge University Press, 1990. Dans un article important, Paul Pierson tire toutes les conséquences politiques d'une telle analyse : «When Effects Becomes Cause : Policy Feedback and Political Change », World Politics, 45 (4), juillet 1993, p. 595-628. Cf. aussi Bruno Palier, Giuliano Bonoli, 
Il s'agit de montrer ici que la « nouvelle politique de l'État providence » qui se manifeste durant la présidence Clinton ne correspond qu'en partie au modèle formulé par Paul Pierson. En effet, le processus de réforme n'est pas entièrement dominé par des stratégies d'évitement. Bien au contraire, la recherche de crédits électoraux demeure un élément essentiel du débat sur les politiques sociales.

Au cours de la présidence Clinton, l'articulation des stratégies d'évitement et de recherche de crédit électoral se transforme en fonction des cycles électoraux et des aléas de la conjoncture économique '. Trois moments caractérisent cette période : 1992-1994, dominé par la débat sur le système de santé et la recherche prudente de crédits électoraux ; 1994-1996, ayant comme trait distinctif l'attitude défensive du président face à la double majorité républicaine au Congrès, manifeste avec l'adoption de la réforme de l'aide sociale ; le second mandat de Bill Clinton, lui, est marqué par l'avènement des surplus budgétaires fédéraux et une recrudescence de la recherche de crédits électoraux inséparable du débat sur l'avenir des retraites. À chacune de ces étapes correspond un type d'articulation des stratégies politiques instrumentalisant le thème du fédéralisme. Celui-ci constitue donc un élément essentiel à la compréhension du débat américain concernant l'avenir des politiques sociales. L'utilisation sélective du thème fédéral permet, le cas échéant, un comportement tactique, voire opportuniste, visant à s'assurer des soutiens tout en désamorçant les critiques. Si le fédéralisme est bien l'une des principales sources de stratégies d'évitement, son emploi n'est en rien unilatéral. Il peut également contribuer, dans certaines circonstances, à la recherche de crédits électoraux propre à tout acteur politique.

\section{LE CONTEXTE DES RÉFORMES SOCIALES DE LA PRÉSIDENCE CLINTON}

En 1992, lors de 1'élection présidentielle, un des thèmes les plus importants de la campagne est la réforme de la protection sociale, question qui trouve sa place dans le cadre d'une interrogation plus large sur la structure fédérale des politiques publiques.

«Phénomène de path dependence et réformes des systèmes de protection sociale », Revue française de science politique, 49 (3), juin 1999, p. 399-420. Ces auteurs critiquent de façon pertinente l'usage unilatéral du concept de path dependency. Suite à l'accumulation de réformes en apparence mineures, les systèmes de protection sociale peuvent se redéployer en empruntant un nouveau «chemin » institutionnel. Pour notre part, il ne s'agit pas ici de rendre compte de l'évolution institutionnelle globale du système américain de protection sociale mais, plutôt, de mettre l'accent sur le lien entre le fédéralisme et les stratégies électorales mobilisées par les acteurs politiques.

1. Au cours des deux mandats de la présidence Clinton (1992-1996, puis 1996-2000), le Congrès est renouvelé quatre fois $(1992,1994,1996,1998)$. Le 103' Congrès (1992-1994) est majoritairement démocrate (258 représentants et 57 sénateurs, contre 176 et 43 pour les républicains). Le suivant passe entièrement aux mains du GOP (Grand Old Party), qui rassemble 230 représentants et 53 sénateurs (contre 204 et 47 pour les partisans du président). La majorité reste républicaine pour le $105^{\circ}$, avec 228 représentants et 55 sénateurs (s'opposant à 206 et 45 démocrates). Enfin, en 1998, l'affaire Lewinski ne modifie que marginalement le rapport de force (majorité républicaine de 223-211, tandis que la composition du Sénat reste identique). Le seul changement est la démission de Newt Gingrich comme Speaker de la Chambre au profit de Denny Hastert. 


\section{Béland, F. Vergniolle de Chantal}

Dans leur livre, Putting People First', Bill Clinton et Al Gore font de la réforme de l'État fédéral une de leurs priorités. La question de la protection sociale apparaît comme un élément majeur de ce programme de réforme ?. Durant la campagne, Clinton promet, par exemple, de mettre un terme à l'aide sociale traditionnelle ( «Ending Welfare as we know it $»)^{3}$. Il y a deux volets à cette proposition. Pour le candidat démocrate, il s'agit d'abord de mettre en œuvre des mesures dites de workfare, censées empêcher une utilisation abusive de l'aide sociale. En obligeant les bénéficiaires à retourner sur le marché du travail, le principal résultat attendu est leur responsabilisation, mais, également, des économies budgétaires. Derrière cette concession à des thèmes traditionnels du Parti républicain, se cache une tactique électorale « centriste » du Parti démocrate pour attirer des électeurs des classes moyennes. Dans la pratique, une fois élu, la réforme du welfare s'efface devant un autre projet, ambitieux, et clairement lié à une stratégie de recherche de crédit auprès de l'électorat traditionnel du Parti démocrate (cols bleus, minorités ethniques, professions intellectuelles) : 1'universalisation de la couverture-maladie.

Au total, si on compare les positions de Clinton sur la réforme de l'aide sociale et celle de l'assurance-maladie, le calcul politique apparaît clairement: s'attirer un maximum de soutien en prenant d'abord une attitude modérée, puis, en capitalisant sur cette tactique, lancer une initiative ambitieuse de recherche de crédits électoraux dans un domaine voisin. L'instrumentalisation est ici assez claire, et typique de la position modérée de Clinton qui, de bien des façons, fait preuve d'un pragmatisme qui confine parfois au cynisme, comme le rappelle le politiste américain Stephen Skowronek dans ses analyses de la présidence américaine ${ }^{4}$.

Mais il reste que l'ambition de ce programme de couverture-maladie universelle est indéniable. À tout le moins si on l'évalue par rapport au contexte général des politiques sociales aux États-Unis ${ }^{5}$. Parmi les pays industrialisés, le système de protection sociale américain est considéré comme une «exception», qui se caractérise par la faible amplitude de la protection offerte et par l'absence d'un régime national d'assurance-maladie ${ }^{6}$.

Pour saisir l'originalité de ce système tout en éclairant le contexte institutionnel des mesures proposées par Clinton, il convient d'explorer deux thèmes essentiels.

1. William Clinton, Albert Gore, Putting People First : How We Can all Change America, New York, Times Books, 1992.

2. Se reporter sur ce point à William A. Galston, Geoffrey L. Tibbetts, « Reinventing Federalism: The Clinton/Gore Program for a New Partnership Among the Federal, State, Local, and Tribal Governments », Publius : The Journal of Federalism, 24 (3), été 1994, p. 2348.

3. Clinton renouvelle sa promesse en janvier 1993 lors du message sur l'état de l'Union (allocution traditionnelle du président pour faire valoir ses principaux objectifs politiques). Cf. aussi Congressional Quarterly, 27 juillet 1996, 30, p. 2116 pour une chronologie des positions prises par Clinton sur la réforme à venir.

4. Stephen Skowronek, The Politics Presidents Make. Leadership from John Adams to Bill Clinton, $2^{*}$ éd., Cambridge, Harvard University Press, 1997 (1 $1^{\text {re }}$ éd. : 1993). Se reporter en particulier à la postface « Bill Clinton's Moment in Political Time ».

5. La plupart des manuels américains de science politique consacrent au moins un chapitre à la question. Pour des analyses plus précises, cf. Walter I. Trattner, From Poor Law to Welfare State. A History of Social Welfare in America, $5^{c}$ éd., New York, Free Press, 1994

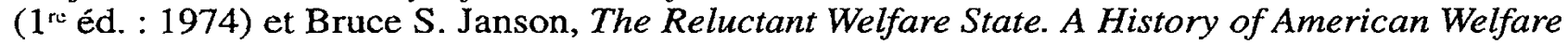
Policies, 2c éd., Pacific Grove, California, Brookes/Cole Publishing, 1993 (1 ${ }^{\text {re }}$ éd. : 1987).

6. Au sujet du débat concernant l'«exception américaine » en matière de protection sociale, cf. Theda Skocpol, Protecting Soldiers and Mothers : The Political Origins of Social Policy in the United States, Cambridge, Harvard University Press, 1992. 
D'abord le considérable mouvement issu du New Deal qui lie centralisation de l'autorité au niveau fédéral, développement des politiques sociales et recherche de crédits électoraux. Ensuite, l'héritage conservateur tel qu'il s'est façonné depuis les années 1960 , et pour lequel l'assistanat et l'extension du pouvoir fédéral sont deux maux à combattre absolument.

Depuis la fin de la guerre de Sécession, la Cour Suprême a freiné l'extension des compétences sociales de l'État fédéral, ainsi que des États fédérés. Sa jurisprudence se base sur une interprétation stricte de la structure fédérale des États-Unis, et, en particulier, de la clause du commerce (article I, section 8), qui définit l'étendue de la capacité d'action commerciale du pouvoir central '. Pour la Cour, à tout le moins jusqu'au New Deal, les pouvoirs publics ne peuvent contrôler l'ensemble du développement économique et social sur la base de cette seule clause constitutionnelle. En effet (pour la Cour de l'époque), cela reviendrait à donner un plein pouvoir d'action au gouvernement fédéral et à ruiner ce qui fait la logique profonde des institutions américaines, leur génie propre, à savoir l'équilibre entre les niveaux de gouvernement ${ }^{2}$.

Avec la politique suivie par Franklin D. Roosevelt, la situation se modifie radicalement. Dans le contexte socio-politique lié à la Crise, les pressions sont telles sur le Judiciaire fédéral, que ce dernier se voit contraint de modifier radicalement sa jurisprudence sur l'étendue du pouvoir commercial du Congrès. La Cour, avec l'arrêt National Labor Relations Board v. Jones and Laughlin Corporation, passe à une interprétation extensive de la clause du commerce, laissant à l'État fédéral la possibilité de mener sa politique de régulation économique et sociale ${ }^{3}$. Le New Deal est donc la première grande étape de constitution de programmes sociaux fédéraux, avec comme point de départ le Social Security Act (1935). Cette loi fondamentale instaure des programmes décentralisés d'aide sociale (Old Age Pensions, Aid to Dependent Children, qui deviendra l'AFDC en 1950) ainsi qu'une cotisation d'assurance-chômage destinée au financement des régimes instaurés par les États fédérés ${ }^{4}$. La pièce

1. Article I, section 8 , « Le Congrès aura le pouvoir... de réglementer le commerce avec les nations étrangères, entre les divers États, et avec les tribus indiennes ». Il faudrait remonter jusqu'à Gibbons v. Ogden (1824, 22 US 1, Chief Justice John Marshall) pour la première utilisation centralisatrice de la clause.

2. Dans I'arrêt Schlechter v. United States de 1935 (295 US 495), qui annule les premières mesures du New Deal, l'opinion majoritaire rédigée par le Chief Justice Charles E. Hughes est tout à fait claire quant à la nécessité de maintenir l'équilibre institutionnel en conservant une interprétation restrictive de la clause du commerce. Pour une présentation d'extraits des principaux arrêts de la Cour suprême en français, cf. Elisabeth Zoller, Grands arrêts de la Cour Suprême des États-Unis, Paris, PUF, 2000 (coll. « Droit fondamental »).

3. Opinion majoritaire rédigée par Charles E. Hughes, 1937 (301 US 1). Bruce Ackerman a donné une interprétation originale de ce renversement en le replaçant dans une théorie juridique dite dualiste où le New Deal peut être caractérisé comme un moment constitutionnel (higher lawmaking period). Lors de ces périodes, la lecture de l'équilibre institutionnel du pays est profondément modifiée, comme ce fut déjà le cas pendant la ratification en 1787 et après la guerre de Sécession. L'intérêt de cette analyse est de prendre en compte la pluralité des logiques - juridiques, mais aussi politiques et sociales - qui président à la gestation des moments constitutionnels. Cf. Bruce Ackerman, Au nom du peuple. Les fondements de la démocratie américaine, Paris, Calmann-Lévy, trad. française : 1998, avec une préface de Patrick Weil.

4. Aujourd'hui encore, les régimes d'assurance-chômage sont administrés par les États fédérés. Le pouvoir central ne joue donc qu'un rôle mineur en matière d'assurance-chômage, ce qui explique son peu de pertinence pour notre propos. Sur la genèse de ce système et, plus généralement, du Social Security Act, on lira Edwin E. Witte, The Development of the Social Security Act, Madison, University of Wisconsin Press, 1962. 
maîtresse du Social Security Act est cependant le régime fédéral d'assurance-vieillesse (Old Age and Survivors Insurance), auquel s'est ajouté en 1956 un régime d'assurance-invalidité (Disability Insurance).

Parallèlement, le mode d'interprétation juridique légitimant l'action de l'État fédéral, va aller s'élargissant jusqu'à atteindre son extension maximum au milieu des années 1960. En fait, depuis 1937, la Cour interprète la clause commerciale de la Constitution d'une manière telle que le commerce entre les États et à l'intérieur de ceux-ci soit de la compétence du pouvoir régulateur du Congrès. De la sorte, il n'est pas, concrètement, une seule activité « sociale » hors de la juridiction du pouvoir central. À ceci s'ajoute, depuis les années 1950 , une jurisprudence constamment en faveur des droits civiques (Civil Rights) qui, en réduisant l'autonomie des États fédérés sur ce point, donne l'impression, surtout dans le Sud, de vider de sa substance la souveraineté étatique '. Traditionnellement, développement économique et protection des libertés fondamentales sont en fait les deux sources auxquelles puise la Cour pour légitimer l'intervention fédérale aux dépens des États. Ce processus s'accélère dans les années 1960. En parallèle, c'est à cette époque que l'État fédéral se lance dans des innovations sociales considérables. Dans le cadre de la Grande Société (Great Society), de nouvelles lois sont votées qui cherchent à lutter contre la discrimination raciale et également à réduire les inégalités sociales ${ }^{2}$.

Ce rapide panorama de l'évolution des politiques sociales aux États-Unis indique clairement la ligne suivie, celle d'une centralisation de l'autorité au niveau fédéral, qui s'effectue au détriment des États fédérés, et qui s'identifie généralement à la gauche de l'échiquier politique. Dans la pratique, cette expansion du rôle de l'État fédéral s'accompagne d'une recherche de crédits électoraux, par laquelle les hommes politiques des deux partis s'efforcent de s'attribuer la responsabilité pour l'augmentation des prestations et la création de nouveaux programmes sociaux ${ }^{3}$. L'élargissement graduel du régime fédéral d'assurance-vieillesse renvoie directement à cette stratégie de recherche de crédit, surtout poursuivie par le Parti démocrate, mais pas uniquement, comme en témoigne l'indexation des prestations par les républicains au début des années $1970^{4}$. L'assimilation politique sociale, centralisation, et recherche de crédits électoraux, dont on trouve les racines dès le mouvement populiste dès années 1890 et qui s'est transmise depuis par Théodore

1. L'arrêt Brown v. Board of Education of Topeka (1954, 347 US 483, rédigé par le Chief Justice Earl Warren) est à l'origine de cette nouvelle jurisprudence.

2. L'année 1964 est marquée par l'adoption de deux lois importantes. La première, que le Président signe le 2 juillet, porte sur les droits civiques. Le Civil Rights Act fait suite à l'arrêt de la Cour Suprême de 1954, à l'amendement constitutionnel de 1964 qui supprime la poll tax et à diverses mesures législatives, toutes dispositions qui visaient à obtenir la disparition des pratiques ségrégationnistes et discriminatoires. Le mois suivant, L. Johnson signe l'Economic Opportunity Act qui comprend des mesures visant à lutter contre la pauvreté. L'année suivante, le Congrès vote le Medicare et le Medicaid, qui instaurent les bases d'un système fédéral de protection médicale, des mesures d'aides aux écoles publiques et aux étudiants de l'enseignement supérieur, une loi sur les droits électoraux, le Voting Rights Act.

3. Sur ce thème, on lira le livre classique de Martha Derthick, Policymaking for Social Security, Washington, Brookings Institution, 1979. L'article de R. K. Weaver constitue un complément théorique incontournable pour cette analyse : "The Politics of Blame Avoidance », art. cité.

4. R. Kent Weaver, Automatic Government : the Politics of Indexation, Washington, Brookings Institution, 1988. 


\section{Politiques sociales et stratégies électorales}

Roosevelt et Franklin D. Roosevelt, trouve son apogée dans les années 1960 avec la Great Society de Lyndon Johnson. Une décennie plus tard, un politiste aussi reconnu que Théodore Lowi pouvait sans crainte évoquer non plus les États-Unis, mais bien l'État Uni lorsqu'il analysait l'évolution du système politique américain '. Ce processus de centralisation est, au moins en partie, lié à une recherche de crédits électoraux de la part de la classe politique fédérale, et tout particulièrement des démocrates.

Dans ce contexte, les opposants conservateurs à la politique de Johnson font de la lutte contre les empiétements du pouvoir fédéral un de leurs thèmes majeurs. Là aussi, le processus enclenché par le New Deal arrive à son terme : $1^{\prime}$ enjeu de la défense des États fédérés contre le pouvoir central passe définitivement dans le camp républicain, celui-là même qui a pourtant mené la guerre contre les États sécessionnistes en 1860-1865. Très exactement un siècle plus tard, dès 1964, Barry Goldwater, candidat républicain à la présidentielle, se lance dans de puissantes dénonciations de l'extension des compétences de l'État central, et prône une défense sourcilleuse des droits des États (States' Rights), terme qui, dans le contexte politique de l'époque, permet le ralliement de tous les conservateurs opposés au Mouvement des droits civiques (Civil Rights Movement) et au développement des politiques sociales fédérales, principalement dans le Sud ${ }^{2}$. Cette campagne marque à bien des égards le début officiel du mouvement conservateur actuel, qui triomphe pour la première fois en 1980, lors de l'élection de Ronald Reagan à la présidence.

Même si ce conservatisme est constitué par une pluralité de tendances idéologiques, il n'en reste pas moins que le combat contre l'extension bureaucratique de l'État fédéral est une de ses constantes, et ce, quelle que soit la mouvance à laquelle on $s^{\prime}$ intéresse ${ }^{3}$. Certes, ce thème est plus ou moins mis en avant selon les conjonctures la campagne de 1968 marque, de ce point de vue, une modération certaine par rapport à celle de 1964 -, mais il est toujours présent. Il est souvent renforcé par l'anticommunisme foncier qui imprègne l'ensemble du mouvement conservateur et sur lequel Ronald Reagan a considérablement capitalisé lors de ses deux mandats, en particulier en politique étrangère, tandis qu'en interne, la volonté de création d'un « Nouveau Fédéralisme » (New Federalism) était un des points forts du premier mandat. En ce qui concerne les politiques sociales, la valorisation du marché, la réduction des dépenses budgétaires et la tentative de réforme de l'aide sociale (welfare) marquent la prési-

1. Théodore J. Lowi, « Europeanization of America ? From United States to United State », dans T. J. Lowi, Alan Stone, Nationalizing Government. Public Policies in America, Berverley Hills, Sage Publications, 1978, p. 15-29.

2. Sur le personnage de B. Goldwater, décédé en 1998, et son action politique, cf. Lee Edwards, Goldwater : The Man Who Made a Revolution, Washington, Regnery Publishing Co., 1995, et Robert Alan Goldberg, Barry Goldwater, New Haven, Yale University Press, 1995. Sur la tactique sudiste du parti républicain depuis les années 1960, l'ouvrage qui, le premier, s'est fait l'écho de cette thèse, est celui de Kevin P. Phillips, The Emerging Republican Majority, New Rochelle, Arlington House, 1969. Pour une analyse récente plus théorique, cf. John Gerring, Party Ideologies in America (1828-1996), Cambridge, Harvard University Press, 1998. En français, on peut se reporter à Denis Lacorne, « La politique du soupçon d'immoralité : comparaisons franco-américaines $»$, Pouvoirs, 63, mars 1993, p. 89-98.

3. Pour une analyse de ces différentes tendances, cf. George H. Nash, The Conservative Intellectual Movement in America since 1945, $2^{\circ}$ éd., Wilmington, Delaware, Intercollegiate

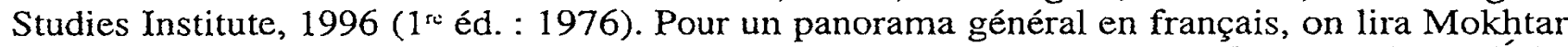
Ben Barka, La nouvelle droite américaine. Des origines à l'affaire Lewinski, $22^{\circ}$ éd., Paris, Édition du Temps, 1999 (1 $1^{\text {rc }}$ éd. : 1996). 


\section{Béland, F. Vergniolle de Chantal}

dence Reagan '. Au cours de cette période, la logique d'austérité budgétaire s'impose. Mais, comme le souligne Paul Pierson, les réformes conservatrices de la protection sociale prônées par Reagan ne sont jamais menées à terme. Face aux attaques des démocrates et à la mobilisation des groupes de bénéficiaires, la présidence poursuit des stratégies d'évitement qui l'incitent à abandonner les projets de réforme les plus conservateurs ${ }^{2}$.

Dans le domaine social comme en matière de politique étrangère, la logique du conservatisme américain est généralement la même : la recherche de l'ennemi intérieur ou extérieur ${ }^{3}$. Comme le souligne George Nash, la disparition de l'ennemi communiste à partir de 1989-1990 a entraîné une réorientation considérable de la pression conservatrice contre l'État fédéral. Ainsi, lors des élections au Congrès de 1994, le programme conservateur inspiré par Newt Gingrich, Le contrat avec l'Amérique, repose sur une dénonciation constante du rôle intrusif de l'État fédéral, considéré comme un Léviathan dangereux pour les libertés fondamentales.

Confronté à ce double héritage, Bill Clinton adopte une attitude modérée, tentant de préserver une partie de la tradition progressiste du Parti démocrate, tout en récupérant une partie du message conservateur. Le pragmatisme de Clinton est ici patent et, derrière 1'habillage « grand public » que constitue le label de « nouveaux démocrates », on trouve clairement le thème développé par Stephen Skowronek d'une politique de «préemption » (preemption), c'est-à-dire une appropriation de certains des thèmes du camp adverse pour s'assurer un soutien populaire le plus large possible et remporter les élections ${ }^{4}$. C'est dans le cadre de cette tactique que l'on peut comprendre l'engagement du président en matière de politique sociale, promettant à la fois de réformer l'aide sociale, et tentant de lancer un vaste programme national d'assurance-maladie. Alors que la réforme de l'aide sociale apparaît comme une stratégie d'évitement susceptible de contrer les accusations de « laxisme moral » formulées par les républicains, le projet concernant l'assurancemaladie s'inscrit dans une stratégie de recherche de crédits électoraux (voir la section suivante).

L'évolution idéologique du Parti démocrate a frappé les observateurs ${ }^{5}$. Concrètement, elle est le résultat des efforts internes du parti pour se réformer idéologiquement et offrir une solution alternative crédible face à un conservatisme au pouvoir et qui devient la référence intellectuelle au sein du champ politique. Les termes du débat

1. Frédéric Lesemann, La politique sociale américaine. Les années Reagan, Paris, Syros/ Alternatives, 1988.

2. Paul Pierson, Dismantling the Welfare State ?..., op. cit.

3. Le parallèle entre anticommunisme et volonté de réforme du fédéralisme a constitué le point de départ de la présidence Reagan. Son slogan de «l'Empire du Mal » pour désigner

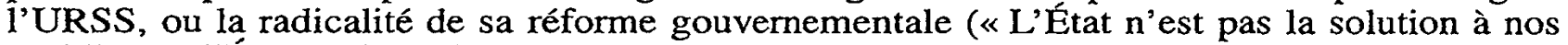
problèmes, l'État est le problème ») sont des mythes fondateurs de la légende reaganienne. Sur la situation récente, on peut renvoyer, à propos du caractère profondément antifédéral de la pensée de Newt Gingrich, à William F. Jr. Connelly, «Newt Gingrich - Professor and Politician : The Anti-Federalist Roots of Newt Gingrich's Thought », Southeastern Political Review, 27 (1), mars 1999, p. 103-129.

4. Pour une brève explication théorique : S. Skowronek, The Politics Presidents Make..., op. cit., p. 43-45.

5. Sur ce point, on peut renvoyer à Nicol C. Rae, Southern Democrats, Oxford, Oxford University Press, 1994. Le livre offre une analyse idéologique de l'évolution du parti dans la période contemporaine. Plus précisément, sur l'action de Clinton au sein du Parti démocrate, cf. Jon F. Hale, « The Making of the New Democrats », Political Science Quarterly, 110 (2), 1995, p. 207-232. 
public sont largement fixés au sein des «boîtes à idées 》 (think tanks) conservatrices, le terme «liberal » est discrédité (on n'évoque plus que le «L-Word»), et les démocrates ont donc, de manière urgente, besoin de se redéfinir une identité politique originale '. Sur le plan organisationnel, c'est là en grande partie l'œuvre du DLC (Democratic Leadership Council), structure informelle au sein du parti et qui, instituée en 1985 par un groupe de jeunes dirigeants démocrates, se fixe comme objectif un recentrage de l'idéologie du parti pour reconquérir l'électorat perdu au profit des républicains ${ }^{2}$. Le positionnement du DLC au sein des instances démocrates et sa stratégie de développement ont été contrariés plusieurs fois, mais au final, malgré - ou peut-être à cause - de sa position initialement floue, le DLC s'est imposé comme un lieu de réflexion incontournable, créant un organisme de recherche lui étant propre (le Progressive Policy Institute), tout en publiant une revue, le New Democrat. Ce succès est dû en grande partie aux échecs répétés du Parti démocrate face à Reagan (1984), puis à Bush senior (1988). La stratégie « libérale » de W. Mondale marque le début de la fin pour l'aile la plus à gauche du parti, tandis qu'à l'inverse la stratégie délibérément «attrape-tout » (catch-all) de Michael Dukakis, a contribué à brouiller son image et, en définitive, explique son échec. Le DLC a ainsi profité d'une « fenêtre d'opportunité » (window of opportunity) à l'intérieur des instances du parti pour non seulement s'implanter, mais aussi évoluer et passer d'un simple forum de discussion à un groupe de travail proposant des perspectives idéologiques nouvelles ${ }^{3}$. C'est au moment de l'ascension fulgurante du DLC, quand ce dernier s'implante au sein des organisations locales et non plus seulement nationales, que Clinton, à la recherche d'une plus grande visibilité institutionnelle, réussit à devenir le premier président du DLC qui ne soit pas de Washington (mai 1990, après le retrait de Gephart). Ce cadre l'a placé au centre des mutations idéologiques du parti. À la fois acteur et agent de ce changement, Clinton a utilisé le DLC comme tremplin pour construire ses réseaux partisans et orienter l'inscription à l'agenda interne ${ }^{4}$, en particulier en ce qui concerne la

1. Sur la prééminence idéologique des conservateurs, notamment au travers de leurs think tanks, cf. Jean Stefancic, No Mercy : How Conservative Think Tanks and Foundations Changed American's Social Agenda, Philadelphie, Temple University Press, 1996 et David M. Ricci, The Transformation of American Politics: The New Washington and the Rise of Think Tanks, New Haven, Yale University Press, 1993. Pour une présentation en français sur un secteur précis, cf. Daniel Béland, « Expertise et politique des retraites. L'influence des think tanks aux États-Unis », L'Année de la régulation, 4, 2000 , p. 32-54.

2. Parmi ceux-ci, on peut citer Richard Gephart (Représentant démocrate du Montana) comme premier président, entouré d'Alvin From (président du Caucus démocrate de la Chambre des Représentants), Sam Nunn (sénateur démocrate de Géorgie), Chuck Robb (Gouverneur démocrate de Virginie), Lawton Chiles (Sénateur démocrate de Floride), Bruce Babbitt (gouverneur démocrate de l'Arizona), et James R. Jones (Représentant de l'Oklahoma) comme porte-parole. Plus tard, des figures aujourd'hui célèbres comme Al Gore et Bill Clinton se sont rapprochées du DLC, en particulier le jeune gouverneur de l'Arkansas.

3. Toute cette évolution est clairement relatée dans Jon F. Hale, « The Making of the New Democrats », art. cité. Cf. aussi Byron E. Shafer, «The Partisan Legacy : Are There any New Democrats ? », dans Colin Campbell, Bert Rockman, The Clinton Legacy, New York, Chatham House, 2000 ; ainsi que Paul S. Herrnson, « Bill Clinton as a Party Politician and Party Leader : the First Term », dans Paul S. Herrnson, Dilys M. Hill, The Clinton Presidency. The First Term, Londres, Macmillan Press, 1999.

4. Le concept d'inscription à l'agenda (agenda-setting) désigne le processus par lequel certaines questions économiques et sociales deviennent des « problèmes » dans le débat public : Frank L. Baumgartner, Brian D. Jones, Agendas and Instability in American Politics, Chicago, University of Chicago Press, 1993 et John W. Kingdon, Agendas, Alternatives and Public Policy, 2 éd., New York, Harper Collins, 1995 (1 ${ }^{\text {rc }}$ éd. : 1984). 
réforme de la protection sociale '. Ce projet, comme on l'a vu, cherche à opérer une synthèse, empirique si ce n'est idéologique. D'une part, Clinton reprend une orientation conservatrice articulée autour de stratégies d'évitement pour alléger les responsabilités de l'État fédéral en matière d'aide sociale et, d'autre part, il tente de se poser en innovateur pour améliorer le fonctionnement global du système social, renouant en cela avec une stratégie politique de recherche de crédits électoraux qui a été le propre du Parti démocrate depuis le New Deal. Cette réalité permet de nuancer considérablement la thèse de P. Pierson, qui voit dans les stratégies d'évitement l'essentiel de la « nouvelle politique de l'État providence ». Aux États-Unis du moins, une telle politique se caractérise par l'imbrication complexe de stratégies d'évitement et de recherche de crédit. Il convient maintenant d'explorer cette imbrication, qui se manifeste tout au long de la présidence Clinton. De la sorte, il sera possible d'éclairer la fonction du fédéralisme dans le débat social qui marque l'Amérique des années 1990. La structure fédérale permet aux acteurs politiques de formuler leurs propositions d'une manière telle qu'ils peuvent à la fois éviter les reproches éventuels qui pourraient leur être adressés, tout en se réservant l'attribution des aspects positifs de la réforme engagée. Autrement dit, l'exemple des politiques sociales sous Clinton montre que le rôle du fédéralisme est parfois ambivalent et ne se réduit pas toujours à un pur et simple alibi inséparable d'une stratégie d'évitement.

\section{LE PROJET CLINTON SUR L'ASSURANCE-MALADIE}

Contrairement à la plupart des pays industrialisés, les États-Unis ne possèdent aucun système national d'assurance-maladie. Les employeurs et les compagnies d'assurances constituent donc les principaux pourvoyeurs de protection médicale ${ }^{2}$. Depuis le milieu des années 1960, l'État fédéral intervient toutefois massivement dans le système de santé américain. Deux programmes essentiels matérialisent l'intervention fédérale dans ce système : le Medicare et le Medicaid. Instauré en 1965, le Medicare protège depuis 1972 l'ensemble des ayants droit des régimes fédéraux d'assurance-vieillesse et $d^{\prime}$ 'assurance-invalidité ${ }^{3}$. Assurance-hospitalisation financée par une cotisation obligatoire, ce programme entièrement centralisé rembourse également, contre le paiement d'une prime mensuelle, une partie des honoraires médicaux. Programme destiné aux assistés sociaux, le Medicaid offre, quant à lui, une aide substantielle aux États fédérés, qui prennent généralement en charge la gestion de l'aide-

1. Le nombre de membres du DLC passe de 219 (1989) à 400 (1991), et jusqu'à 700 (1992). Son influence interne croît dans les mêmes proportions. Quant aux idées du DLC, on peut les trouver dans, William Galston, Elaine C. Karmack, «The Politics of Evasion : Democrats and the Presidency », Washington, Progressive Policy Institute, 1989.

2. Pour une présentation commode du système de santé américain, cf. Elisabeth Chamorand, Le système de santé américain. Poids du passé et perspectives, Paris, La Documentation française, 1996. Cf. aussi John J. Dillulio Jr, Richard R. Nathan, Making Health Care Reform Work: The View From the States, Washington, Brookings, 1994.

3. Au sujet de la genèse du Medicare, cf. Peter A. Corning, The Evolution of Medicare... from Idea to Law, Washington, United States Government Printing Office (US Department of Health, Education, and Welfare, Social Security Administration, Office of Research and Statistics, Research Report $n^{\circ}$ 29), 1969 et Theodore R. Marmor, The Politics of Medicare, Chicago, Aldine Publishing Co., 1973. 
médicale . Par l'entremise des programmes Medicare et Medicaid, les pouvoirs publics exercent donc une fonction « résiduelle » dans le système de santé américain 2 . Ils interviennent en effet directement auprès des seules populations «vulnérables » (retraités, assistés sociaux), qui sont généralement mal protégées par les entreprises et les compagnies d'assurances. Quant à ces dernières, elles prennent en charge la majorité - mais non la totalité - des salariés.

À la veille des élections présidentielles de 1992, le débat social américain porte sur les lacunes de ce système fragmenté, qui repose sur la complémentarité apparente des secteurs privé et public. La lacune la plus évidente concerne le nombre important $\mathrm{d}$ 'individus qui sont privés d'assurance-maladie. Comme elle se trouve fréquemment liée à l'emploi, la mise à pied du salarié le prive généralement de ce type de protection. Au début des années 1990, la montée du chômage provoque d'ailleurs une inquiétante hausse du nombre d'individus sans assurance : en 1992, environ $15 \%$ de la population américaine ne possède aucune assurance-maladie. Quant aux dépenses médicales des familles, elles augmentent à un rythme accéléré. Entre 1987 et 1992, ces dernières passent en moyenne de $15,1 \%$ à $18,2 \%$ du revenu familial ${ }^{3}$. Dans ce contexte, les syndicats et les coalitions de consommateurs se mobilisent pour réclamer une protection médicale plus adéquate. Retransmises par les médias, ces revendications incitent la classe politique à examiner la question de la couverture-maladie à partir de la fin des années $1980^{4}$.

Du point de vue des responsables politiques et des experts fédéraux, le problème du plafonnement des dépenses de santé paraît tout aussi fondamental. Malgré une couverture-maladie perfectible, le système américain de santé se révèle en effet des plus coûteux. Représentant plus de 13,5\% du PNB en 1992, les dépenses de santé ne cessent d'augmenter, ce qui constituerait une menace pour la compétitivité internationale de l'économie américaine ${ }^{5}$. Dès lors, le Congrès ainsi que les assemblées législatives

1. Les États fédérés continuent également à jouer un rôle essentiel dans la régulation du système médical américain : épidémiologie, gestion des hôpitaux publics, réglementation de la profession médicale et des compagnies d'assurances, leurs attributions demeurent nombreuses. A ce sujet, cf. Éveline Thévenard, «Fédéralisme et système de santé : initiatives locales de réforme de l'assurance-maladie », Revue française d'études américaines, 77, juin 1998, p. 88102 (notamment p. 88-90).

2. D'abord formulée par Titmuss, la notion de « résiduel » se trouve développée dans un ouvrage déjà classique : Gosta Esping-Andersen, Les trois mondes de l'État-providence. Essai sur le capitalisme moderne, Paris, PUF, trad. française 1999 (1 $1^{\mathrm{re}}$ éd. : 1990). Cette notion désigne le rôle dominant du marché dans la protection sociale. Dans un système « résiduel » de protection sociale, les pouvoirs publics s'efforcent généralement de compléter la prévoyance individuelle et la protection offerte par le secteur privé. Pour ce faire, ils offrent un minimum de subsistance aux plus démunis tout en assurant certaines catégories sociales «à risque », qui ne peuvent bénéficier d'une couverture assurancielle privée.

3. E. Chamorand, Le système de santé américain, op. cit., p. 114.

4. En 1991, la victoire surprise - lors d'une élection partielle - du candidat démocrate au Sénat, Harris Wofford, favorise directement l'inscription à l'agenda de la question de la réforme du système de santé. Donné perdant au début de la campagne, Wofford remporte la victoire grâce à une campagne axée sur la question de la couverture-maladie universelle. Après sa victoire, l'ensemble de la classe politique se sent dans l'obligation de prendre position sur cette question : Jacob S. Hacker, « National Health Care Reform: An Idea whose Time Came and Went », Journal of Health Politics, Policy and Law, 21 (4), hiver 1996, p. 647-696.

5. Les dépenses de santé réelles per capita ont augmenté de 4,4\% entre 1980 et 1990 ; au cours de la décennie précédente, cette hausse n'avait été que de $3,8 \%:$ Joseph P. Newhouse, «Medical Care Costs : How Much Welfare Loss? », Journal of Economic Perspectives, 6, été 1992, p. 3-21. 


\section{Béland, F. Vergniolle de Chantal}

étatiques envisagent des mesures susceptibles de limiter la hausse des dépenses nationales de santé tout en assurant une meilleure protection médicale à la population ' Dans ce contexte, la question de l'assurance-maladie occupe une place fondamentale dans la campagne présidentielle de 1992, dans laquelle le candidat Clinton accuse le président républicain George Bush de ne rien faire pour résoudre les problèmes du système de santé américain ${ }^{2}$. À ce moment, l'inscription à l'agenda de la question de la réforme du système atteint son point culminant. Cette réforme apparaît d'ailleurs comme le grand projet du premier mandat de Clinton. Pour lui, il s'agit de rechercher des crédits électoraux auprès des franges les plus défavorisées de la population active (dont le couverture-maladie est précaire ou inexistante), qui constituent une clientèle électorale traditionnelle pour le Parti démocrate. Dans le domaine de la santé, la recherche de crédits électoraux - plutôt que les stratégies d'évitement - se situe au cœur de la campagne présidentielle clintonienne.

Pour profiter de l'élan suscité par sa victoire de novembre 1992, le nouveau président s'efforce de lancer «sa » réforme au début de son mandat. Selon une loi bien connue des hommes politiques américains, le soutien accordé aux nouveaux présidents amorce en effet son déclin quelques mois seulement après leur entrée en fonction. La «lune de miel» post-électorale représente ainsi une occasion unique mais brève de passer à l'histoire ${ }^{3}$. Esquissé en 1992 mais dévoilé officiellement le 22 septembre $1993^{4}$, le projet Clinton (Health Security) s'inscrit dans un modèle de la « concurrence contrôlée » (managed competition), qui s'oriente vers une régulation complexe du capitalisme médical américain ${ }^{5}$. Résultat d'un compromis idéologique et politique susceptible de rallier une partie de l'électorat républicain, le projet Clinton repose sur trois impératifs en apparence contradictoires ${ }^{6}$. En plus de l'impératif de couverture-maladie universelle, ses concepteurs admettent en effet la nécessité de préserver la libre concurrence et celle de réduire les dépenses nationales de santé ainsi que la taille du déficit budgétaire fédéral. Inséparable de la stratégie centriste poursuivie par Clinton, son projet renvoie à une tentative d'augmenter la protection sociale sans heurter trop directement les intérêts du capitalisme médical. En ce qui concerne la réforme du système de santé, la recherche de crédits électoraux (universalisation de la

1. Frank Baumgartner, Jeffrey C. Talbert, «From Setting a National Agenda to Making Decisions in Congress », Journal of Health Politics, Policy and Law, 20 (2), été 1995, p. 437445. À ce moment, des expériences sociales orientées vers la réalisation de ce double objectif ont déjà été lancées dans des États fédérés comme l'Oregon et Hawaï: E. Thévenard, « Fédéralisme et système de santé », art. cité.

2. Theda Skocpol, Boomerang: Clinton's Health Security Effort and the Turn against Government in U.S. Politics, New York, W. W. Norton, 1996, p. 36-38.

3. Lyndon Johnson a exprimé en des termes simples cette « loi » implicite de la vie politique américaine : « Je ne cesse de me battre car je sais que cette lune de miel ne durera pas. Chaque jour je perds un peu de mon capital politique. C'est pourquoi nous devons continuer, ne jamais abandonner. » (Johnson cité dans Jack Valenti, A Very Human President, New York, W. W. Norton, 1975, p. 144).

4. «Clinton's Health Plan : Transcript of President's Address to Congress on Health Care », New York Times, 23 septembre 1993, p. A24.

5. Au sujet de l'inscription à l'agenda de la « concurrence contrôlée », on lira Jacob Hacker, The Road to Nowhere : The Genesis of President Clinton's Plan for Health Security, Princeton, Princeton University Press, 1997.

6. Pour une présentation de ce plan, cf. François Sellier, « L'échec du projet Clinton d'assurance maladie ", Revue française des affaires sociales, 1995, 2/3, p. 237-257. Outre les ouvrages de Hacker et Skocpol déjà cités, on consultera également Nicholas Laham, A Lost Cause : Bill Clinton's Campaign for National Health, Westport, Praeger, 1996. 
couverture-maladie) cohabite avec trois stratégies d'évitement chargées de réduire les risques électoraux auxquels s'exposent la présidence.

La réforme Clinton représente en effet une importante source de risques électoraux. Organisés en puissants groupes de pression, les médecins ainsi que les représentants du capitalisme médical (propriétaires d'hôpitaux, industries pharmaceutiques) voient d'un mauvais œil toute intrusion fédérale susceptible de réduire leur liberté et, surtout, leurs marges de profit. La puissance de ces groupes est à l'origine d'une première stratégie d'évitement : la reconnaissance du rôle central du marché dans le cadre de la réforme Clinton. Pour ménager les groupes d'intérêt issus du capitalisme médical, les conseillers du président - avec à leur tête sa femme Hilary - imaginent en effet un système dans lequel l'intervention accrue de l'État fédéral pourrait se conjuguer avec le maintien de la compétition entre les assureurs et entre les pourvoyeurs de soins. Tout en garantissant une couverture-maladie à l'ensemble de la population, ce projet s'efforce de conserver la « liberté du choix » actuellement offerte au « consommateur » de soins médicaux. Plus important encore, le projet Clinton se trouve orienté par un impératif de contrôle budgétaire hérité de l'ère Reagan. À ce sujet, 1'objectif de la présidence s'affirme clairement : plafonner les dépenses nationales de santé, entre autres en imposant certaines règles susceptibles de contrôler l'augmentation des primes d'assurance '. Pour ne pas se faire accuser d' « irresponsabilité budgétaire », la présidence s'efforce de lier universalisation de la couverture-maladie et maîtrise des dépenses de santé. Il s'agit là de la deuxième stratégie d'évitement inséparable du projet Clinton.

L'examen attentif de ce projet révèle également que, en plus de reconnaître les principes de l'économie de marché, il accorde une place centrale au rôle des États fédérés. Chargés de mettre en place les organismes d'affiliation des individus et des entreprises (health alliances), ces derniers exerceraient une fonction administrative essentielle dans un réseau décentralisé de régulation. Dans le rapport officiel chargé de faire la promotion de ce projet, on s'efforce d'ailleurs de présenter l'État fédéral comme un simple instigateur, qui s'effacera rapidement une fois la réforme mise en place. À ce moment, le «peuple», les milieux d'affaires ainsi que le secteur privé réguleront le système de santé en fonction de leurs choix ${ }^{2}$. Face à l'image négative de la bureaucratie fédérale véhiculée depuis la fin des années 1960 par la droite conservatrice, les réformateurs démocrates eux-mêmes tentent de minimiser le rôle pourtant essentiel que jouerait l'État fédéral dans le système de régulation proposé. Cet effacement proclamé de l'État fédéral - et la reprise du discours anti-bureaucratique de la droite conservatrice - constitue la troisième et dernière stratégie d'évitement inséparable du projet Clinton, qui vise la recherche de crédit auprès de l'électorat démocrate traditionnel tout en neutralisant les éventuelles critiques du Parti républicain et des groupes de pression associés au capitalisme médical.

Malgré ces trois stratégies d'évitement, le projet Clinton suscite la vive opposition des milieux d'affaires et des élus républicains. Plus important encore, l'objection des compagnies d'assurances et de l'Association médicale américaine ainsi que l'attitude de plus en plus hostile des employeurs menace directement le projet. D'abord hésitante, la Chambre de Commerce devient par exemple - à la suite des pressions exercées par ses membres républicains - un farouche adversaire de ce projet ${ }^{3}$. Peut-

1. Par exemple : E. Chamorand, Le système de santé américain, op. cit., p. 119.

2. Health Security: The President's Health Care Plan, octobre 1993, p. 8-9, brochure citée dans T. Skocpol, Boomerang, op. cit., p. 124.

3. T. Skocpol, Boomerang, op. cit., p. 158. 


\section{Béland, F. Vergniolle de Chantal}

être en raison de la campagne organisée notamment par les élus et les médias conservateurs, le projet Clinton se trouve également discrédité auprès de la population. Déjà divisée, elle manifeste des craintes quant à la détérioration des soins que pourrait provoquer l'accomplissement de cette réforme '. Malgré le soutien de l'AFL-CIO, la levée de boucliers suscitée par le projet Clinton incite plusieurs membres influents du Congrès à le rejeter. Au lieu d'apporter des modifications au texte législatif proposé par la présidence, deux des cinq comités législatifs chargés de l'examiner (l'Énergie et le Commerce à la Chambre, le Comité des Finances au Sénat) le rejettent avant même que l'ensemble des élus puissent voter. Malgré les efforts de négociation de la présidence, le projet de loi est donc abandonné avant même la tenue des élections législatives de novembre $1994^{2}$. Après l'échec des trois stratégies d'évitement mentionnées ci-dessus, le projet Clinton se transforme en véritable fiasco politique.

La défaite de ce projet conduit le président et les autres élus démocrates à adopter une attitude prudente en matière d'assurance-maladie. Dans la seconde moitié des années 1990, l'universalisation de la couverture-maladie ne fait donc plus partie de l'agenda législatif fédéral. Tout au long de cette période, la réforme du programme Medicare - et, plus généralement, l'idée de contrôle des coûts du système de santé demeure toutefois au centre du débat politique. À coup d'ajustements budgétaires en apparence modestes, le Congrès s'efforce ainsi, tout au long de la présidence Clinton, de contrôler la hausse des dépenses liées à ce programme ${ }^{3}$. Parce que ce dernier concerne essentiellement les personnes âgées, les prévisions au sujet du vieillissement démographique alimentent d'ailleurs les craintes de la population et du législateur ${ }^{4}$. Profitant de ces inquiétudes, certains républicains et leurs alliés des think tanks conservateurs formulent des projets de réforme plus importants, qui s'orientent soit vers la restructuration complète du Medicare, soit vers une réduction importante de la protection offerte par ce programme. Jusqu'à ce jour, ces projets sont toutefois demeurés lettre morte. Le programme Medicare reste populaire auprès des personnes âgées et toute réforme drastique pourrait conduire à une levée de boucliers porteuse d'énormes risques électoraux pour les responsables politiques ${ }^{5}$.

1. Pour une analyse de l'évolution des sondages d'opinion en 1993-1994, cf. Mollyann Brodie, Robert J. Blendon, « The Public's Contribution to Congressional Gridlock on Health Care Reform », Journal of Health Politics, Policy and Law, 20 (2), été 1995, p. 402-410.

2. F. Sellier, «L'échec du projet Clinton d'assurance maladie », art. cité, p. 251.

3. Après des négociations entre la Présidence et le Congrès, ces ajustements sont généralement intégrés aux accords budgétaires annuels. C'est dans le cadre de l'accord budgétaire de l'année 1997 que les ajustements les plus significatifs sont adoptés. Fort techniques, ils diversifient les options offertes aux ayants droit tout en réduisant certains des remboursements offerts : Marilyn Moon, Barbara Gage, Alison Evans, An Examination of Key Medicare Provisions in the Balanced Budget Act of 1997, Washington, Urban Institute, septembre 1997.

4. Pour une analyse complète de la situation de ce programme, cf. Marilyn Moon, Medicare Now and in the Future, 2 "éd., Washington, Urban Institute Press, 1997 ainsi que Robert D. Reischauer et al. (dir.), Medicare: Preparing for the Challenges of the 21st Century, Washington, National Academy of Social Insurance, 1998.

5. Au sujet des différentes propositions en présence, cf. Marilyn Moon, Restructuring Medicare: Impacts on Beneficiaries, Washington, Urban Institute, janvier 1999. Face à la modestie des projets de réforme fédéraux, les États fédérés s'efforcent de prendre l'initiative pour accomplir les deux objectifs principaux de la défunte réforme Clinton : le contrôle des dépenses de santé et l'universalisation de la couverture-maladie. Devant les campagnes lancées par les puissants groupes de pression du capitalisme médical, les législateurs étatiques paraissent toutefois particulièrement vulnérables. Comme leurs homologues fédéraux, ils poursuivent diverses stratégies d'évitement, qui les confinent souvent à l'inaction. Sur ces questions, cf. E. Thévenard, « Fédéralisme et système de santé », art. cité, p. 92 et 99. 
Le projet Clinton et, dans une moindre mesure, la politique de réforme du Medicare, témoignent de l'imbrication des stratégies d'évitement et de la recherche de crédits électoraux dans le domaine de la protection médicale. En cette matière, le thème du fédéralisme se trouve mobilisé de manière essentiellement pragmatique. Si la recherche de crédit électoral amène le président à faire sienne l'universalisation de la couverture-maladie, il se garde de formuler des propositions qui mettent l'accent sur la mission régulatrice de l'administration fédérale. L'idée de « concurrence régulée » atteste de l'usage ambigu et instrumental du thème du fédéralisme dans le débat social américain. L'État fédéral - par l'intermédiaire du Président - serait là pour garantir une couverture-maladie universelle. La réalisation de cette réforme ne s'accompagnerait toutefois d'aucun renforcement de l'administration fédérale, qui laisserait une grande latitude aux État fédérés ainsi qu'aux acteurs économiques. Ainsi, la centralisation (couverture-médicale universelle imposée par l'État fédéral) est inséparable de la recherche de crédits électoraux alors que le thème de la décentralisation sert de stratégie d'évitement. Ce lien entre décentralisation et stratégies d'évitement s'exprime clairement dans la réforme de l'aide sociale de 1996. Avant d'analyser cette réforme, soulignons que l'instrumentalisation du thème fédéral est tout sauf univoque. Par exemple, son emploi judicieux lors du débat au sujet de la couverture-médicale universelle aurait pu permettre de faciliter l'adoption du projet Clinton. Il aurait donc facilité la recherche de crédits électoraux nécessaires au président démocrate. L'échec du projet Clinton ouvre la voie à l'utilisation la plus fréquente du thème fédéral, consistant à en faire un alibi pour des réformes conservatrices porteuses de nombreux risques électoraux. La loi sur l'aide sociale de 1996 résume parfaitement ce mode d'action.

\section{DES ÉLECTIONS LÉGISLATIVES À LA RÉFORME DE L'AIDE SOCIALE DE 1996}

Pas un seul observateur n'a sous-estimé l'impact des élections de 1994 au Congrès. Comme l'indique Jean-Pierre Lassale, cette échéance fait office de véritable séisme dans le paysage politique américain '. Pour la première fois depuis 1954 , les deux Chambres sont dirigées par le Parti républicain. Par ailleurs, la génération qui arrive au pouvoir est fortement mobilisée autour d'une idéologie conservatrice rendue publique dans un programme électoral, Le Contrat avec l'Amérique. Le Speaker de la Chambre des représentants, Newt Gingrich, entend bien mener une politique agressive de confrontation avec la Présidence pour mettre en œuvre les principales mesures contenues dans le programme républicain ${ }^{2}$. L'impératif d'austérité budgétaire qui

1. Jean-Pierre Lassale, « Les élections intermédiaires de 1994 aux États-Unis : révolution conservatrice ou nouveau populisme », Revue de droit public et de science politique, 4, 1995, juillet-août, p. 863-881.

2. Le Contrat avec l'Amérique met en avant dix mesures de réforme qui, par leur ampleur, ne sont ni plus ni moins qu'une remise en cause du double héritage du New Deal et de la Great Society. On y trouve les propositions suivantes : établir une obligation d'équilibre budgétaire, assurer la sécurité publique, réformer l'aide sociale, renforcer la famille, réformer l'assurancevieillesse, renforcer la politique de défense, baisser les impôts sur les particuliers et les entreprises, adapter le système juridique à la massification des contentieux, et limiter le mandat des membres du Congrès. Cf. aussi « Detours Ahead as GOP Drives to Reshape Government », Congressional Quaterly, 45, 19 novembre 1994, p. 3331-3346. 
domine la vie politique américaine depuis l'ère Reagan devient incontournable. Pour les conservateurs, cet impératif prend une forme antifédérale.

L'influence considérable des élections législatives de 1994 sur la présidence Clinton se fait tout particulièrement sentir en matière de politiques sociales. L'orientation modérée de la campagne de 1992 est plus que renforcée et le champ des politiques sociales est celui dans lequel la tactique d'appropriation des thèmes conservateurs menée par Clinton s'affirme le plus. Le 22 août 1996, le président signe le Personal Responsibility and Work Opportunity Reconciliation Act, qui constitue une réforme majeure de l'aide sociale en démantelant le programme AFDC (Aid to Families with Dependent Children) et en transférant un grand nombre de responsabilités en matière sociale aux États fédérés '. L'évolution du président sur ce thème est néanmoins sensible dès les premier signes d'enlisement de la réforme de l'assurancemaladie, et même avant, comme semble l'indiquer son bilan en tant que Gouverneur de l'Arkansas. Clinton va rapidement comprendre que ce champ est celui dans lequel le recentrage démocrate doit être poussé le plus loin. Ce positionnement doit clairement se révèler payant électoralement. Déjà en tant que simple Gouverneur, Clinton avait adopté une politique des plus surprenantes pour un élu démocrate. Au sein de la National Governors' Association (NGA), il ouvre pour soutenir l'élaboration du Family Support Act (1988), mesure phare de l'administration Reagan dans le domaine de la réforme de l'aide sociale ${ }^{2}$. Lors de son élection, il se positionne au centre de l'échiquier politique par sa promesse de réformer l'aide sociale. Ce thème est dès lors un des aspects essentiels du programme des Nouveaux Démocrates, dans la mesure où son utilisation tactique devrait permettre de gagner le soutien des classes moyennes et de l'électorat des banlieues aisées. Tout en refusant de signer les projets de loi les plus durs au sujet du démantèlement de l'aide sociale (il met son veto à une première version en décembre 1995, puis encore une fois en janvier 1996), il laisse la porte ouverte à certains accommodements ${ }^{3}$. Ainsi, lorsque la NGA propose au début de 1996 une nouvelle version, plus modérée, de réforme de l'aide sociale, Clinton peut aisément la qualifier d'encourageante. La voie vers l'acceptation de la réforme est tracée durant l'été. Cet épisode est capital pour l'évolution de la stratégie politique du Président. Son glissement à droite se renforce. En 1996, Clinton accepte dans les faits l'essentiel des positions conservatrices tout en réduisant considérablement le poids des thèmes démocrates traditionnels. En prévision des élections présidentielles de

1. Sur cette loi (Public Law 104-193), on lira Daniel Béland, « La fin du Welfare State : de la guerre contre la pauvreté à la guerre contre les pauvres », Esprit, 232, mai 1997, p. 38-58. On peut aussi renvoyer à «After 60 Years, Most Control Is Passing to States », Congressional Quaterly, 31, 3 août 1996, p. 2190-2196. Les principales dispositions légales sont disponibles dans un numéro ultérieur du Congressional Quaterly: «Welfare Overhaul Law ", 21 septembre 1996, p. 2696-2705. Pour une évaluation de l'impact de cette loi, cf. Sheryll D. Cashin, « Federalism, Welfare Reform, and the Minority Poor : Accounting for the Tyranny of State Majoritỳ », Columbia Law Review, 99 (3), avril 1999, p. 552-627.

2. La loi (Public Law 100-485), qui apparaissait extrêmement sévère à l'époque, obtient néanmoins un fort soutien bipartisan au Congrès. Elle met l'accent sur des programmes sociaux et/ou éducatifs qui, en devenant obligatoires pour les bénéficiaires de 1'aide sociale, doivent leur faciliter la recherche d'un emploi. À ce sujet, cf. F. Lesemann, La politique sociale américaine, op. cit.

3. En janvier 1996, après avoir refusé la seconde proposition du Congrès, il déclare cependant, dans son Discours sur l'État de l'Union, qu'il est prêt à signer tout plan bipartisan de réforme que lui proposerait le législatif. Plus tard, en mai de la même année, il fait l'éloge d'une réforme de l'aide sociale menée par le Wisconsin et dont l'orientation est nettement conservatrice. 
novembre 1996, le Président s'efforce de poursuivre ce qu'on pourrait qualifier de double stratégie d'évitement. Tout en soutenant une réforme conservatrice pour neutraliser les accusations de «laxisme », il promet de pallier les lacunes de la nouvelle loi après sa réélection, et ce, pour prévenir les accusations de « trahison » qui émanent de son propre parti. Autrement dit, les positions démocrates sont réduites à une portion congrue devant ce nouvel impératif que Clinton s'efforce de faire passer : la réélection prime sur l'idéologie. Une stratégie frontale vis-à-vis des républicains conduirait à l'impasse au vu du rapport de force politique du moment. L'objectif de la tactique clintonienne est clairement l'élection de 1996 qui, outre une poursuite de la présidence démocrate pourrait éventuellement voir la fin de la domination républicaine au Congrès.

Si l'impact de la loi de réforme de l'aide sociale est si profond, c'est que les modifications qu'elle introduit sont drastiques. Suivant en cela l'exemple de réformes conservatrices menées par certains États fédérés, l'État fédéral limite considérablement les possibilités d'aide aux plus démunis, en argumentant autour de l'idée de responsabilité individuelle, sans oublier la nécessité d'obtenir de substantielles réductions budgétaires '.

Le principal aspect de cette loi est le transfert complet de compétences en matière d'aide sociale de l'État fédéral aux États fédérés. C'est tout particulièrement le cas pour le programme $A F D C$ (Aid to Families with Dependent Children), qui d'ailleurs, perd son appellation d'origine pour répondre dorénavant au sigle TANF (Temporary Aid to Needy Families). L'AFDC constituait d'ailleurs une source de polémique idéologique depuis la fin des années 1960 , et ce, en raison de l'augmentation du nombre des bénéficiaires (essentiellement des mères-célibataires dont un grand nombre sont d'origine afro-américaine) ${ }^{2}$. En ce sens, la signature de cette réforme couronne près de trente ans de mobilisation conservatrice contre l'aide sociale, et particulièrement contre le programme $A F D C$ qui se trouvait accusé d'encourager l'augmentation du nombre d'enfants nés hors mariage (out-of-wedlock children), phénomène qui touche principalement les familles afro-américaines. Ce programme, qui reprenait en les systématisant une série d'initiatives étatiques, avait été créé en 1935 par le Social Security Act. Il visait à assister les familles monoparentales dans le besoin. Sa gestion était confiée aux États fédérés. Ce sont eux qui définissaient le niveau des besoins. Ils établissaient aussi les critères d'admissibilité ainsi que le montant de l'aide à apporter, le tout devant respecter les contraintes fixées par l'État fédéral. Washington contribuait au financement du système proportionnellement à l'effort fourni par chaque État fédéré. Les montants versés étaient automatiquement reconduits d'une année sur l'autre et ce, quel que soit le nombre de bénéfi-

1. Faire des États fédérés des lieux d'expérimentation pour le développement ultérieur de politiques nationales est un des avantages du fédéralisme par rapport à un système unitaire. Il est constamment mis en avant par les théoriciens du fédéralisme, comme, par exemple, David Osborne, à l'origine du projet Clinton de réforme de l'administration (NPR, National Performance Review), Laboratories of Democracy, Boston, Harvard Business School Press, 1988. À l'origine, on doit au juge Louis Brandeis dans son opinion dissidente (dissent) de New State Ice Co. v. Liebmann (1932, 285 US 262), d'avoir formulé cette image de l'État fédéré comme un laboratoire d'innovation.

2. L'augmentation du nombre de bénéficiaires est notamment liée aux campagnes en faveur de la dèségrégation de l'aide sociale menée dans le Sud. Quant aux «problèmes » de la famille afro-américaine, ils sont devenus un thème du débat politique américain à la suite de la publication en 1965 du fameux «rapport Moynihan »: «The Negro Family : The Case of National Action ». Sur ces questions, cf. F. Lesemann, La politique sociale aux États-Unis, op. cit. 
ciaires dont la situation personnelle donne droit aux prestations (entitlement program). Toute modification en la matière supposait en fait une intervention législative du Congrès.

Si l'AFDC était déjà largement délégué aux États fédérés dans sa gestion, il est, depuis 1996, entièrement de leur responsabilité, dans la mesure où il leur incombe de définir les critères d'admissibilité des prestataires, sans supervision fédérale '. L'État fédéral se contente de distribuer des financements «en bloc» (on parle de block grant), en se basant sur la somme que les États consacraient au programme avant l'adoption de la réforme ${ }^{2}$. Le rôle de l'État fédéral est donc relativement limité si on le compare avec son action antérieure. En revanche, il l'est beaucoup moins si l'on prend en considération toute une série de nouvelles obligations d'obédience conservatrices, créées par la loi, et qui, au final, donnent à l'État fédéral une forte capacité de coercition. En effet, si Washington ne fixe plus les critères d'admissibilité et s'il réduit bien son financement, il peut cependant imposer des règles très strictes, notamment en ce qui concerne l'incitation au travail (workfare), second volet de la loi après la décentralisation (devolution) ${ }^{3}$. Ainsi, quelle que soit l'attitude adoptée au niveau fédéré, tout adulte qui touche des prestations d'aide sociale doit obligatoirement commencer à travailler dans les deux ans qui suivent le début de sa réception des prestations. Un objectif chiffré en la matière est imposé aux États : de $25 \%$ en 1997, on doit passer à $50 \%$ des bénéficiaires de l'aide sociale en 2002 qui doivent retourner sur le marché du travail, et ainsi quitter les listes d'aide sociale (welfare rolls). En cas d'échec, les États concernés voient les fonds fédéraux diminuer de $5 \%$, et ainsi de suite chaque année jusqu'à un maximum de $21 \%$ de l'aide fédérale en moins. Les autres mesures contenues dans la loi sont, elles aussi, éminemment restrictives. La plus importante est incontestablement la limite temporelle imposée aux prestataires, et ce, pour la durée de leur existence. Le plafond est d'une durée de cinq ans. En cas de dépassement, elles sont tout simplement supprimées : l'ex-prestataire ne peut plus compter que sur luimême. Par ailleurs, les immigrants illégaux ne sont plus admissibles à l'aide sociale. De même, le programme fédéral de bons d'alimentation (Food Stamps) ${ }^{4}$, est, lui aussi, accompagné d'exigences sévères, notamment en termes de travail. Les conditions et la durée d'admissibilité sont réduites de façon plus ou moins importante selon les catégories. Par exemple, les chômeurs sans enfants, âgés de 18 à 50 ans, ne pourront rece-

1. Concrètement, les États peuvent déterminer qui est admissible aux prestations, de quelle forme d'aide il s'agit (argent liquide ou service social), sa durée exacte, et les termes/ conditions régissant son attribution. En ce sens, le programme cesse d'être un entitlement, c'est-à-dire un programme où ceux qui remplissent les critères d'admissibilité deviennent automatiquement bénéficiaires de l'aide sociale.

2. Au sujet des «block grants», se reporter par exemple à Ronald F. King, "Welfare Reform, Block Grants, Expenditure Caps, and the Paradox of the Food Stamps Program », Political Science Quarterly, 114 (3), automne 1999, p. 359-886.

3. Depuis la fin des années 1960 , le terme workfare qualifie les mesures d'incitation - ou d'obligation - au travail susceptibles de mettre fin à la « dépendance » envers l'aide sociale. L'idée de la responsabilisation par le travail est donc à la base des mesures de workfare. On lira à ce sujet l'article de Sylvie Morel, « France et États-Unis : les politiques d" "insertion" et de workfare en matière d'aide sociale », Interventions économiques, 27, printemps 1996, p. 47-75.

4. Ce programme a été lancé par le président Kennedy en 1961. Ce n'est qu'en 1964 qu'il devient véritablement un programme fédéral, alors qu'il n'était auparavant qu'à un stade expérimental. Les ménages à faible revenu reçoivent des coupons qu'ils peuvent échanger contre des aliments. Le programme est encadré par l'État fédéral et les États fédérés, qui ne peuvent prendre la moindre intiative en la matière et sont simplement chargés de la gestion courante. Ainsi, les conditions d'admissibilité sont fixées par le pouvoir central. 
voir ces bons que pour une durée cumulative de trois mois pour chaque séquence de trois ans où ils seront admissibles à ce programme. D'autres dimensions soulignent encore cet aspect conservateur de la loi. Les mères adolescentes (non mariées) ne pourront recevoir de prestations que si elles poursuivent des études tout en demeurant chez leurs parents. Par ailleurs, une mère seule est obligée de collaborer avec les autorités dans le but de retrouver le père de son ou de ses enfant(s) afin de le forcer à contribuer financièrement à leur éducation. Si elle refuse de coopérer, elle verra ses prestations réduites dans des proportions importantes '. La double logique qui préside à cette réforme - morale avec la lutte contre la dépendance et la réaffirmation du travail, mais aussi budgétaire, dans la mesure où le transfert aux États réduit la charge financière du budget fédéral - répond aux aspirations des conservateurs et permet par ailleurs à Clinton de capitaliser sur son acceptation de la loi pour se rapprocher de l'électorat centriste ${ }^{2}$. Après la signature d'une telle réforme, il semble plus difficile pour les républicains de l'accuser de « laxisme».

Ces profondes modifications de l'aide sociale aux États-Unis sont toutefois farouchement critiquées, non seulement sur une base idéologique, mais aussi pour des raisons théoriques ${ }^{3}$. Dans une perspective purement partisane, de nombreux groupes au sein du parti démocrate voient dans la décision de Clinton, un reniement des valeurs profondes du Parti, notamment en ce qui concerne la protection des minorités ethniques qui sont les principales bénéficiaires de l'aide sociale. Les opposants à la réforme se recrutent donc dans les rangs du Congressional Black Caucus et de son équivalent hispanique, auxquels il faut ajouter différents groupes, plus marginaux (Progressive Caucus, par exemple) ${ }^{4}$. Mais l'échec de ces différents mouvements face aux « Nouveaux démocrates » relativise considérablement leur audience ${ }^{5}$.

Ce qui caractérise cette loi c'est donc une instrumentalisation certaine du thème fédéral, mais une instrumentalisation qui va dans une seule et même direction, celle de la baisse des dépenses sociales de l'État fédéral. La rhétorique antifédérale employée par les conservateurs au sujet de la décentralisation des politiques publiques apparaît comme une stratégie d'évitement permettant de masquer les conséquences sociales les plus néfastes des réductions budgétaires. L'alibi de la décentralisation et de la responsabilisation des États permet de rendre les réductions budgétaires drastiques moins visibles, tout en diminuant la responsabilité de la classe politique fédérale en la matière. Cette stratégie d'évitement rejoint tout à fait le diagnostic porté par Martha Derthick, lorsqu'elle attribue au fédéralisme le triste privilège de faciliter la

1. D. Béland, « La fin du Welfare State », art. cité.

2. Pour des compte rendus de la stratégie électorale de Clinton, cf. C. Campbell, B. Rockman, The Clinton Legacy, op. cit, ainsi que P. Herrnson, D. Hill, The Clinton Presidency, op. cit.

3. Par exemple, sur les oppositions internes au Parti démocrate: «Welfare Overhaul Forces Ready To Start without Clinton », Congressional Quaterly, 13, avril 1994, p. 800-803. Beaucoup plus universitaire, mais pas moins virulent, voir le bilan critique établi par $\mathrm{S}$. Cashin, «Federalism, Welfare Reform, and the Minority Poor », art. cité.

4. N. Rae (Southern Democrats, op. cit.) donne une typologie des rapports de force au sein du Parti démocrate en faisant une distinction entre les New Liberals, frange la plus à gauche, puis les regulars, héritiers du New Deal, et enfin les néolibéraux, centristes (dont Clinton est un des représentants), et les élus sudistes.

5. En fait, l'essentiel de la critique faite à la loi sur l'aide sociale porte sur la question du nivellement par le bas (race to the bottom) qu'elle induit en matière de protection sociale. Dans une logique purement fonctionnaliste, il semble qu'à partir du moment où capital et travail circulent sans entraves sur un territoire donné, les investissements se font là où le coût de la maind'œuvre est le plus faible. 
confusion des lignes de responsabilité démocratique. Selon elle, la structure fédérale porte en son sein le risque suivant : permettre aux acteurs politiques d'un niveau de gouvernement (par exemple, les États fédérés) de se décharger de leurs responsabilités sur d'autres acteurs, situés à un niveau différent (par exemple, l'État fédéral). Le flou relatif qui en résulte permettrait une conduite purement opportuniste de la part des élus. Elle se ferait au détriment des citoyens, peu capables de déterminer qui est, en dernier ressort, à l'origine de telle ou telle mesure. Cet argument est actuellement repris par la jurisprudence récente de la Cour suprême sur le fédéralisme. La Cour s'inspire largement de cette idée d'opacité pour appeler à un tri, une clarification des compétences entre les différents niveaux de gouvernement. C'est le cas en particulier avec l'arrêt New York v. United States de 1992, où l'opinion majoritaire rédigée par la juge O'Connor annule une régulation fédérale en matière de déchets radioactifs. L'argument de ce juge repose sur une critique de la confusion de responsabilités politiques (accountability) induite par la mise en œuvre de ladite régulation. La relative opacité du système fédéral pour l'électeur est une des réalités les plus courantes - et, paradoxalement, les moins étudiées - de la vie politique américaine. Concrètement, ce phénomène se retrouve dans la multiplication des stratégies d'évitement. Ces stratégies concernent autant l'inscription à l'agenda que la mise en ouvre des politiques publiques '.

Une grande partie du débat sur la réforme de l'aide sociale s'est construit, sur la thématique de la dévolution, du retour de compétences vers les États fédérés, et ce, d'autant plus facilement que lesdits États ont été pionniers dans la tentative de réforme de l'aide sociale. L'utilisation du thème de la décentralisation est un puissant facteur de légitimation pour n'importe quelle politique en général, mais tout particulièrement dans le domaine de l'aide sociale ${ }^{2}$. De ce point de vue, proposer de transformer le programme $A F D C$ pour lui faire quitter son statut mécanique (entitlement) et le financer en bloc (block grant), s'apparente à une utilisation cynique de la rhétorique de la décentralisation qui est, selon Cashin, une constante dans la justification de l'emploi croissant de ce mode de financement fixe : «L'histoire récente des propositions de financement fédéral en bloc est une preuve de plus de la tendance, parmi les responsables politiques nationaux, consistant à mettre en avant les valeurs du fédéralisme pour décentraliser les politiques sociales, sans faire attention aux différences très réelles en termes institutionnels et politiques entre États fédérés et État fédéral ${ }^{3}$ ». Le souci principal des responsables conservateurs, dans cette optique, n'est certainement pas le respect de l'équilibre fédéral, mais bien plutôt, la baisse de la charge budgétaire

1. Ce thème de la relative opacité du système fédéral remonte aux débats de ratification de la Constitution en 1787. Les opposants au projet de Philadelphie y voyaient une preuve de son caractère aristocratique. Actuellement, les travaux de science politique mettent l'accent sur la pratique politique d'évitement. Outre les références déjà citées tout au long du texte, on peut renvoyer, sur ce point précis du lien avec la structure fédérale, à Martha Derthick, « Up-To-Date in Kansas City : Reflections on American Federalism », PS : Political Science \& Politics, 25 (4), décembre 1992, p. 671-675. Se reporter aussi à l'arrêt New York v. United States, 1992, O'Connor, 505 US 144, ou encore à Printz v. United States, 1997, Scalia, 521 US 898. Sur le poids de l'antifédéralisme dans le conservatisme actuel, cf. François Vergniolle de Chantal, «Le sentiment antigouvernemental aux États-Unis: essai de généalogie », Esprit, 269, novembre 2000 , p. 119-135.

2. Pour plus de détails, on lira S. Cashin, « Federalism, Welfare Reform, and the Mirority Poor », art. cité, surtout p. 566-574. Tout un appareil de sondage est disponible pour confirmer cet argument. On peut les trouver dans John D. Donahue, Disunited States, New York, Basic Books, 1997. Ils confirment que les États sont couramment perçus comme de meilleurs gestionnaires que l'État fédéral et ce, dans un grand nombre de domaines, incluant la question sociale.

3. Cf. S. Cashin, « Federalism, Welfare Reform, and the Minority Poor », art. cité, p. 570. 


\section{Politiques sociales et stratégies électorales}

que représente l'aide sociale, ainsi qu'une volonté d'en finir avec les politiques « libérales » (au sens américain) instituées depuis le New Deal !

L'impératif de décentralisation se manifeste clairement lorsqu'il s'agit de limiter la compétence de l'État fédéral en matière budgétaire. Il n'en est plus du tout de même en ce qui concerne l'aspect « moral » de la loi, strictement conservateur, et visant à responsabiliser les bénéficiaires de l'aide sociale. C'est là une seconde preuve de la pure et simple instrumentalisation du thème de la décentralisation : s'il peut apparaître dans le débat pour légitimer tel ou tel aspect du projet, il disparaît aussitôt quand il pourrait aller contre des valeurs conservatrices plus substantielles, comme la responsabilité individuelle, l'éthique du travail et le respect des valeurs familiales. Lorsque la loi de 1996 aborde ces problèmes, c'est l'État fédéral qui, au contraire, est en charge de les résoudre. C'est là d'ailleurs une très grande innovation, dans la mesure où les questions de cet ordre sont traditionnellement laissées aux États fédérés, le pouvoir central se contentant, lui, d'intervenir de façon tout à fait périphérique ${ }^{2}$. Les normes fédérales conservatrices sont extrêmement contraignantes et encadrent les États pour les orienter définitivement vers des politiques restrictives vis-à-vis des plus défavorisés. Les États sont sans doute libres d'aller plus loin dans la réduction des prestations, mais on peut douter de la possibilité de développer un vaste programme « libéral », étant donné l'orientation qui est imposée à leur propre système de protection sociale. Autrement dit, l'action conservatrice se caractérise par une attitude paradoxale qui voit la coexistence d'une réforme décentralisée de l'aide sociale dont le but quasi explicite est la réduction du poids budgétaire de l'État fédéral, et, par ailleurs, une centralisation, morale cette fois, et non plus budgétaire, par le biais de mécanismes comme le workfare et la limitation de la durée d'admissibilité ${ }^{3}$.

1. La réforme de l'aide sociale en 1996 marque en fait la troisième des tentatives conservatrices d'établir un « Nouveau Fédéralisme » (New Federalism) après celles de R. Nixon et de $R$. Reagan. Toutes deux répondaient à des objectifs qui n'étaient pas uniquement motivés par « l'harmonie » du fédéralisme. Pour Nixon, il s'agissait d'abord et avant tout de rationaliser la gestion de la multiplicité de programmes mis en marche par Lyndon Johnson. Instituer des financement en bloc revenait à réduire l'emprise que certains groupes d'intérêts avaient sur des programmes catégoriels, plus limités dans leur objectif, et, de ce fait, plus susceptibles d'être capturés par des intérêts particuliers. Reagan a repris cette tactique avec une volonté supplémentaire, celle de réduire la taille des appareils étatiques à tous les niveaux du pays (politique dite de retrenchment) car, dans les faits, le financement en bloc s'apparente le plus souvent à une réduction des fonds fédéraux mis à disposition. Pour plus de détails sur ces politiques, on consultera Timothy Conlan, From New Federalism to Devolution. Twenty Five Years of Intergovernmental Reform, Washington, Brookings Institution, 1998.

2. Sur cette division des tâches entre substantif (mœurs, religion, droit civil, etc.), laissé aux États fédérés, et procédural (fixation de limites et/ou de conditions de déroulement), attribué à l'État fédéral, on lira 'Théodore Lowi, «Avant le conservatisme et au-delà », Revue française de science politique, 32 (2), avril 1990, p. 76-103. Sur le paradoxe de la politique menée par le 104" Congrès, cf. «GOP Confounds Expectations, Expands Federal Authority », Congressional Quarterly, 44 (2), novembre 1996, p. 3117-3122.

3. La première option correspond aux idées de conservateurs radicaux comme Charles Murray, auteur de Losing Ground: American Social Policy (1950-1980), New York, Basic Books, 1994, pamphlet prônant, entre autres, le démantèlement complet de l'aide sociale. L'autre option serait présentée dans le livre de George F. Will, Statecraft as Soulcraft. What Government Does, New York, Simon and Schuster, 1983. L'intervention de l'État fédéral est présentée comme une nécessité pour garantir un minimum de cohésion sociale, de justice, voire de vitalité dans le pays. Bien loin d'une position minimaliste, cette attitude semble être un avatar hamiltonien au sein d'un conservatisme qui se présente pourtant de plus en plus sous un jour antifédéral. 
Au total, si l'on cherche à évaluer l'impact du fédéralisme dans la réforme sociale menée sous la présidence Clinton, on est conduit à conclure sur l'ambiguïté de son rôle. De 1994 à 1996, la question du retour de pouvoir aux États fédérés a été particulièrement présente, comme jamais depuis le début de la présidence Reagan. Presque tous les acteurs politiques se sont faits les avocats de la dévolution, que ce soit dans le camp démocrate ou républicain. Ce simple constat montre, si besoin en est, le degré de légitimité auquel les arguments conservateurs sont parvenus '. C'est autour de leur position que s'effectue l'essentiel du débat, et les positions adverses sont comme inexistantes. En ce sens, l'ensemble du déroulement de la réforme de 1'aide sociale peut être présentée comme une victoire conservatrice pour l'inscription à l'agenda public de leurs idées. Mais, et sans doute pour la même raison, on peut souligner que le fédéralisme a un rôle instrumental dans la légitimation des idées conservatrices. Comme l'indiquent tous les sondages depuis des années, la population est favorable à un transfert de compétences vers les États fédérés en matière sociale, et c'est sur cette orientation générale que les républicains capitalisent pour assurer la popularité de leurs mesures. En ce sens, la prégnance généralisée du fédéralisme dans le débat public de l'époque, ne permet pas de l'analyser comme un argument substantiel dans le cadre de la réforme de l'aide sociale. Il est utilisé tactiquement pour assurer la domination de thèmes conservateurs plus substantifs (responsabilité, famille, etc.).

Plus important encore, le fédéralisme s'inscrit dans une stratégie politique qui combine recherche de crédit et stratégie d'évitement. Sur le plan moral, les conservateurs peuvent revendiquer leur responsabilité en soulignant que l'État fédéral impose désormais des valeurs morales qui s'inscrivent dans la tradition sociale américaine (éthique du travail, responsabilité individuelle). Les réductions budgétaires contenues dans la loi sont, elles, volontairement masquées par un discours axé autour des idées de décentralisation et de responsabilisation des États. À l'avenir, cette plus grande décentralisation devrait permettre aux conservateurs de rejeter d'éventuelles accusations concernant les lacunes potentielles de l'aide sociale en renvoyant les critiques aux États fédérés. Du point de vue du Parti républicain, la réforme de 1996 combine adroitement stratégies d'évitement et recherche de crédit. Le thème de la dévolution est inséparable des stratégies d'évitement conservatrices.

Sur un plan plus strictement politique, il semblerait que Clinton ait pu capitaliser sur son acceptation de la réforme de 1996 - résultant d'une stratégie d'évitement longuement planifiée - pour s'assurer le soutien des franges modérées de l'électorat, et ce, d'autant plus facilement que les excès du Speaker de la Chambre des Représentants, Newt Gingrich, effraient ces dernières ${ }^{2}$. Clinton apparaît donc comme un candidat du « juste milieu », à l'écoute des aspirations de la classe moyenne. Ainsi, dans

1. La seule voie discordante qui ait un peu d'impact (on ne tient pas compte ici de figures politiques secondaires, présentes au sein du Parti démocrate), a été celle de la secrétaire d'État à la Santé de Clinton, Donna E. Shalala, qui déclare le 28 février 1996 devant le Comité des Finances du Sénat, que les initiatives présentées jusqu'ici par le Congrès et la NGA en matière de réforme sociale ne sont pas admissibles car elles donnent trop de pouvoir aux États. On peut ici remarquer que le problème n'est pas le transfert de compétence en lui-même, mais simplement son étendue.

2. L'agressivité de la stratégie de Gingrich concernant le financement de l'État fédéral a conduit, par deux fois, entre 1994 et 1996, à la suspension de certains services publics. Cette tactique a desservi le Speaker de la Chambre dans le mesure où le Président réussi à lui en faire porter entièrement la responsabilité. Sur ce point, cf. James W. Ceaser, Andrew Busch, Losing to Win: The 1996 Elections and American Politics, New York, Rowman and Littlefield, 1997, p. 44-45. 
un contexte économique favorable, il n'a guère de difficulté à battre son adversaire républicain, le sénateur Bob Dole '. Lors de son second mandat, il va réorienter sa stratégie en poursuivant une politique de recherche de crédit dans le domaine principal d'intervention de l'État fédéral: l'assurance-vieillesse. Le débat sur l'avenir des retraites est le troisième et dernier temps de la réforme des politiques sociales sous la présidence Clinton.

\section{LE DÉBAT SUR L'AVENIR DES RETRAITES}

Depuis le New Deal, l'État fédéral joue un rôle essentiel dans le domaine des retraites. Instauré dans le cadre du Social Security Act de 1935, le régime fédéral d'assurance-vieillesse constitue en effet le socle sur lequel repose le système de retraite américain ${ }^{2}$. Contrairement à ce qui se produit dans le domaine de la santé, l'intervention de l'État fédéral n'a ici rien de «résiduelle». Dans le champ des retraites, c'est en effet le secteur privé - à travers l'épargne individuelle et les régimes complémentaires - qui fait simplement compléter les mesures fédérales, et non l'inverse. Financé par une cotisation égale des salariés et des employeurs, le régime fédéral d'assurance-vieillesse concerne d'ailleurs plus de $95 \%$ des salariés, à qui il offre un taux de remplacement moyen d'environ $40 \%$ du salaire plafonné ${ }^{3}$. Dans ce cadre, il constitue à la fois l'élément essentiel du système de retraite et du système fédéral de protection sociale.

Depuis la seconde moitié des années 1970, l'avenir budgétaire de ce régime par répartition soulève toutefois de nombreuses interrogations. Malgré le « succès » de la réforme de 1983, qui a rétabli l'équilibre budgétaire de la caisse fédérale, le vieillissement démographique ainsi que l'altération du ratio actifs/inactifs paraissent menacer l'intégrité budgétaire à long terme du régime d'assurance-vieillesse ${ }^{4}$. Bien qu'aucune «crise budgétaire » ne soit prévue avant l'horizon 2030, le débat sur l'avenir des retraites occupe une place essentielle dans la vie politique américaine après la réélection de Clinton en novembre 1996. Loin de porter uniquement sur d'éventuels ajustements budgétaires, ce débat concerne la «privatisation » possible du régime fédéral d'assurance-vieillesse, c'est-à-dire son remplacement - total ou partiel - par des comptes individuels d'épargne. À la suite d'une campagne lancée par les think tanks

1. Clinton gagne l'élection de 1996 avec $49 \%$ du suffrage populaire, alors que Bob Dole n'en totalise que $41 \%$ (Perot, lui, n'obtient que $9 \%$ ). Clinton obtient donc de meilleurs résultats qu'en 1992. À cette époque, il avait remporté l'élection avec seulement $43 \%$ des voix, face à George Bush ( $37 \%$ ) et Ross Perot $(19 \%)$.

2. Complexe et fragmenté, le système de retraite américain se divise en fait en quatre éléments principaux : 1) le régime fédéral d'assurance-vieillesse, qui est l'équivalent du régime de base français ; 2) le programme fédéral d'aide sociale pour les invalides et les personnes âgées dans le besoin (SSI);3) les régimes d'entreprise à prestations définies (defined benefits plans), qui versent des pensions complémentaires à une minorité seulement des salariés américains ;4) l'épargne-retraite individuelle, qu'elle soit encadrée ou pas par l'entreprise. Pour une présentation synthétique, cf. Daniel Béland, «L'avenir des retraites aux États-Unis : défi démographique, débat politique $»$, Futuribles, 244, juillet-août 1999, p. 83-107.

3. Aux États-Unis, le taux de remplacement est inversement proportionnel au salaire. Les salariés les moins nantis bénéficient donc davantage de ce régime.

4. À ce sujet, on consultera le plus récent rapport officiel de l'administration fédérale : « 1999 Annual Report of the Board of Trustees of the Federal Old-Age and Survivors Insurance and Disability Insurance Trust Funds », Washington, 30 mars 1999. 


\section{Béland, F. Vergniolle de Chantal}

conservateurs et leurs alliés républicains, le thème de la « privatisation » du régime fédéral devient en effet un élément essentiel du débat sur l'avenir des retraites. Stimulés par la progression de l'épargne salariale et par les performances exceptionnelles des marchés boursiers dans les années 1990, les opposants à ce régime soutiennent qu'une telle réforme pourrait offrir des rentes plus généreuses aux futurs retraités tout en éliminant les difficultés posées par le vieillissement démographique. Généralement ils s'inspirent donc d'une logique financière inséparable d'un recours massif à l'épargne salariale '. Pour les tenants de cette logique, les salariés devraient épargner pour garantir leur avenir et, indirectement, pour augmenter le taux national d'épargne.

Les questions de l'assurance-maladie et de l'aide sociale occupent l'avant-scène du débat politique durant le premier mandat présidentiel de Bill Clinton. Amorcé dès la fin des années 1970 , le processus d'inscription à l'agenda de la « privatisation »du régime fédéral atteint donc son point culminant après les élections présidentielles de novembre 1996. Une fois la réforme du welfare accomplie, les conservateurs peuvent en effet concentrer leurs efforts sur la question des retraites.

Publié en janvier 1997, le rapport du dernier Conseil consultatif sur la Sécurité sociale (Advisory Council on Social Security) favorise directement l'inscription à l'agenda du thème de la « privatisation » du régime fédéral d'assurance-vieillesse ${ }^{2}$. Incapables de forger un consensus, les douze membres de cette commission d'experts proposent trois projets de réforme distincts, qui seraient susceptibles de résoudre durablement les problèmes budgétaires liés au vieillissement démographique. Parmi ces trois projets, le plus médiatisé s'avère sans conteste le Personal Security Account Plan ${ }^{3}$. En bref, ce projet de réforme prévoit le remplacement graduel du régime d'assurance-vieillesse par des comptes individuels d'épargne ainsi qu'un minimun-vieillesse qui équivaut à deux tiers du taux officiel de pauvreté ${ }^{4}$. S'il était ratifié, ce projet représenterait une rupture fondamentale par rapport au modèle assuranciel à la base du régime fédéral actuel. Au lieu d'offrir des pensions dont le montant se trouve défini à l'avance par des critères actuariels stables (defined benefits), les comptes individuels ne prévoient que le niveau des cotisations (defined contribution), sans garantir celui de la rente offerte. Cette dernière dépendrait en effet des choix d'investissement des individus et, en dernière analyse, des fluctuations des marchés boursiers.

1. D'abord esquissé par des auteurs néolibéraux comme Milton Friedman, cette logique se trouve notamment formulée dans un rapport de la Banque mondiale, publié en 1994 : Averting the Old Age Crisis : Policies to Protect the Old and Promote Growth, Oxford, Oxford University Press, 1994. Au sujet de la logique financière contemporaine, on lira Jill Quadagno: «Creating a Capital Investment Welfare State : the New American Exceptionalism », American Sociological Review, 64 (1), février 1999, p. 1-11.

2. Les conseils consultatifs sont des commissions d'étude composées notamment d'universitaires et de représentants syndicaux et patronaux.

3. Un autre projet de réforme - soutenu par 6 des 13 membres du Conseil consultatif prévoit cependant la consolidation du régime fédéral d'assurance-vieillesse : «Report of the 1994-1996 Advisory Council on Social Security, vol. 1 : Findings and Recommendations », Washington, United States Government Printing Office, 1997.

4. Le système de retraite imaginé par les auteurs de ce plan se rapproche du modèle des « trois piliers » formulé dans le rapport de 1994 de la Banque mondiale : Averting the Old Age Crisis, op. cit. En vertu de ce modèle, les systèmes de retraite devraient reposer essentiellement sur l'épargne obligatoire et, dans une moindre mesure, l'épargne volontaire. Le troisième « pilier » de ce système est l'aide sociale, qui se trouve chargée d'offrir un revenu de subsistance aux individus qui ne peuvent épargner suffisamment pour survivre. 
Depuis 1997, des propositions similaires sont formulées par les think tanks conservateurs et certains membres républicains du Congrès '. Au-delà de leur diversité apparente, ces projets de «privatisation » prônent tous le passage - total ou partiel de la répartition à l'épargne obligatoire, qui serait généralement encadrée par l'État fédéral. Une telle réforme transformerait donc le rôle de l'État fédéral dans le domaine des retraites. Au lieu de garantir un revenu stable à la vaste majorité des retraités, il deviendrait un simple intermédiaire financier entre les cotisants-investisseurs et les marchés boursiers. Pour ne pas effrayer un électorat traditionnellement attaché au régime d'assurance-vieillesse, les conservateurs proposent généralement une « privatisation partielle », c'est-à-dire le détournement d'une partie seulement des cotisations vers des comptes individuels d'épargne.

L'idée d'épargne obligatoire inséparable du thème de la «privatisation » renvoie à une reconnaissance implicite - et paradoxale - du rôle de l'État fédéral, qui se chargerait de garantir l'obligation légale et, dans bien des cas, d'administrer les comptes individuels ${ }^{2}$. La réforme des retraites envisagée par les partisans de l'épargne obligatoire se distingue donc de la réforme de l'aide sociale adoptée en 1996. Au lieu de réclamer la décentralisation du régime fédéral d'assurancevieillesse, ceux-ci soutiennent en effet le développement d'une logique financière dans un cadre institutionnel centralisé, qui s'inscrit parfaitement dans la continuité du Social Security Act de 1935. Le régime d'assurance-vieillesse devrait graduellement céder sa place à une nouvelle mesure fédérale, qui se confondrait toutefois avec l'impératif financier. Bien que le terme «privatisation » soit excessif pour désigner une telle réforme ${ }^{3}$, il évoque néanmoins clairement l'un des objectifs de ses partisans : la satisfaction de la demande d'épargne individuelle du capitalisme financier, qui soutient généralement les efforts des opposants au régime fédéral d'assurance-vieillesse ${ }^{4}$. Les projets de «privatisation» des retraites attestent une fois

1. Pour un inventaire pratique des récents projets de réforme, cf. Henry J. Aaron, Robert D. Reischauer, Countdown to Reform: The Great Social Security Debate, New York, Century Foundation Press, 1998, p. 117-147.

2. Parmi les principaux partisans de la «privatisation» des retraites, seul l'économiste Milton Friedman souhaite le démantèlement pur et simple du régime fédéral - et son remplacement par un plan d'épargne volontaire : Milton Friedman, « Social Security Chimeras », New York Times, 11 janvier 1999, p. A21. Pour Friedman, l'obligation légale serait inacceptable; l'épargne devrait être volontaire. À la fin des années 1990, cette position semble relativement marginale, car même la majorité des élus du Parti républicain soutiennent le recours à l'épargne obligatoire. Cette attitude s'explique sans doute par la volonté d'assurer une augmentation rapide de l'épargne salariale, ce qui ferait l'affaire des institutions financières. Derrière cette logique économique, il serait également possible d'identifier un certain «paternalisme » conservateur, qui s'exprime également dans la réforme de l'aide sociale de 1996. La « privatisation » des retraites généraliserait en effet l'épargne individuelle, hissée au rang de vertu par les conservateurs.

3. Le concept de privatisation implique un retrait radical de l'État: Harvey Feigenbaum, Jeffrey, Chris Hamnett, Shrinking the State: The Political Underpinnings of Privatization, Cambridge, Cambridge University Press, 1999. Comme le souligne S. Teles, le passage à l'épargne obligatoire impliquerait également une plus grande régulation politique (fédérale) des marchés boursiers, comme cela s'est produit en Grande-Bretagne durant les années 1990 : Steven Teles, «Pensions, Social Citizenship and the Ubiquity of the State : the Relevance of the British Privatization Experience in the United States and beyond », Workshop on Social Policy and Devolution, Brandeis University Center for German and European Studies, mars 1999.

4. La logique financière qui sous-tend les projets de «privatisation » se trouve décrite par Lucy ApRoberts, «Salariat et retraite aux États-Unis », thèse de sciences économiques, université Paris I, 1999. 


\section{Béland, F. Vergniolle de Chantal}

encore de l'attitude pragmatique des conservateurs américains envers l'État fédéral. Comme pour la réforme de l'aide sociale, ils entendent placer la centralisation au service d'une valeur morale (responsabilité individuelle) ou de l'intérêt économique de certains de leurs alliés politiques.

Défendus par de nombreux élus républicains ainsi que par quelques « Nouveaux Démocrates » (par exemple, le Sénateur Daniel Patrick Moynihan), les projets de «privatisation partielle » du régime fédéral sont cependant loin de faire l'unanimité. D'après les défenseurs de ce régime, qui se recrutent généralement au sein du Parti démocrate, les partisans de la « privatisation » exagèrent nettement les bienfaits de l'investissement individuel et les futurs effets du vieillissement démographique sur la répartition. En fait, 1'adoption d'un ensemble d'ajustements modestes - détournement des surplus budgétaires fédéraux vers un fonds de réserve, augmentation de la proportion du salaire touchée par les cotisations, modification du système d'indexation automatique - serait susceptible de préserver durablement la solvabilité du régime fédéral, qui représenterait une source de sécurité très supérieure à celle offerte par l'épargne obligatoire ${ }^{1}$.

Pour garantir son avenir budgétaire, certains défenseurs du régime fédéral d'assurance-vieillesse (par exemple, Henry Aaron et Robert Ball) proposent également d'investir une partie de son fonds de réserve sur les marchés boursiers. Actuellement, les surplus de la caisse sont «empruntés » par le trésor fédéral en échange de bons du trésor offrant un faible rendement. En investissant ceux-ci, on augmenterait les revenus de cette caisse sans devoir recourir à une forte hausse du taux de cotisation. Dans son discours sur l'état de l'Union de janvier 1999, le président Clinton a soutenu publiquement cette option en proposant d'investir une partie du fonds de réserve de la caisse fédérale sur les marchés boursiers ${ }^{2}$. Qui plus est, il a proposé le transfert, au cours des quinze prochaines années, de $60 \%$ des surplus budgétaires anticipés vers cette caisse pour augmenter la taille du fonds de réserve. En investissant une partie de ces sommes supplémentaires, il entend réduire le déficit anticipé de la caisse, et ce en profitant du taux de rendement élevé des marchés boursiers pour augmenter ses recettes.

Le détournement des surplus budgétaires fédéraux vers le fonds de réserve s'inscrit dans une stratégie démocrate visant à dissuader la double majorité républicaine au Congrès de voter une importante baisse d'impôt. Plus important encore, l'idée de détourner une partie des surplus fédéraux vers la caisse d'assurance-vieillesse représente une recherche de crédit explicite. En proposant un usage « social » des surplus budgétaires, Bill Clinton se présente comme le « sauveur » du régime fédéral d'assurance-vieillesse. Dans le domaine social, l'avènement des surplus budgétaires fédéraux peut donc créer une remarquable « fenêtre d'opportunité » pour la recherche explicite de crédits électoraux, et ce, tant pour la présidence que pour l'ensemble de la classe politique fédérale. Après deux décennies d'austérité budgétaire et de stratégies d'évitement, les surplus fédéraux apparaissent comme une occasion inespérée pour les deux partis politiques, qui comptent les distribuer pour gagner de nou-

1. Ancien Commissaire à la Sécurité sociale et membre du dernier Conseil consultatif, Robert M. Ball apparaît aujourd'hui comme le défenseur le plus en vue de la répartition. Dans un ouvrage récent, il propose une série d'ajustements modestes pour préserver le régime fédéral : Straight Talk about Social Security: An Analysis of the Issues in the Current Debate, New York, Century Foundation Press, 1998. De tels ajustements reçoivent généralement le soutien des démocrates.

2. «President William Jefferson Clinton State of the Union Address », The White House, Office of the Press Secretaty, 19 janvier 1999. 
veaux'. Alors que les républicains soutiennent généralement d'importantes baisses d'impôt, les démocrates mettent l'accent sur la préservation des régimes fédéraux d'assurance sociale (assurance-vieillesse, Medicare), sans parler de la création de nouvelles politiques publiques ${ }^{2}$. D'un point de vue démocrate, les surplus budgétaires apparaissent comme une source potentielle de crédits électoraux, associés depuis le New Deal à la création de nouveaux programmes sociaux et à l'élargissement des mesures existantes.

Fidèle à sa stratégie « centriste», le président démocrate tend néanmoins une perche aux républicains en proposant, en guise de compromis, l'instauration d'un plan fédéral d'épargne volontaire pouvant compléter, et non remplacer, l'actuel régime d'assurance-vieillesse. Susceptible de satisfaire les économistes en augmentant le taux d'épargne nationale, ce plan complémentaire serait subventionné par le trésor fédéral pour encourager la participation des moins nantis. Reprenant à son compte la logique financière contemporaine, Bill Clinton entend lui aussi mettre l'État fédéral au service des marchés boursiers.

Avec ce projet de réforme en apparence modeste, le président propose - paradoxalement - une extension de la mission économique et sociale de l'État fédéral. En investissant les surplus budgétaires fédéraux sur les marchés boursiers, ce dernier deviendrait un acteur économique de premier plan. Bien que les investissements seraient réalisés par une commission d'experts indépendante, il n'en demeure pas moins qu'une nouvelle mission économique serait confiée à l'État fédéral, dans une société où le rôle économique direct de celui-ci se révèle généralement limité. De plus, l'instauration d'un plan d'épargne complémentaire élargirait directement la place de l'État fédéral dans le domaine des retraites. En plus d'organiser le régime d'assurancevieillesse et l'aide sociale offerte aux personnes âgées, il mettrait en place un système de protection complémentaire, qui serait justifié par la nécessité d'augmenter l'épargne nationale. Malgré sa modération apparente, le projet Clinton sur les retraites semble donc porteur d'un nouvel élargissement de la mission socio-économique de l'État fédéral héritée du New Deal. Plus que jamais, l'État fédéral jouerait un rôle prépondérant dans le système américain de retraite et, plus particulièrement, dans le domaine des politiques publiques destinées aux personnes âgées ${ }^{3}$. Comme durant l'après-guerre, ce projet lie recherche de crédits électoraux et centralisation du système de protection sociale. Clinton s'approprie à nouveau un thème conservateur (l'épargne individuelle), cette fois pour rechercher des crédits électoraux tout en favorisant un élargissement de la mission sociale de l'État fédéral.

En dépit de l'habileté du président, ses propositions suscitent la controverse. Aux yeux des conservateurs, la gestion centralisée des investissements substantiels effec-

1. En raison d'une forte croissance économique (4\% en 1999), le budget fédéral atteint 99 milliards de dollars de surplus pour l'exercice clôt au $1^{\text {er }}$ septembre 1999 (142 milliards d'excédent prévu pour l'exercice 2000-2001). En 1999, les dépenses de l'État fédéral représentent d'ailleurs seulement 18,5\% du PIB, le pourcentage le plus bas depuis 1966. Les experts fédéraux font miroiter un possible surplus budgétaire d'environ 5470 milliards de dollars au cours des 15 prochaines années : Jacques Isnard, «Bill Clinton promet aux États-Unis un avenir sans dette ni déficit », Le Monde, 2 août 1999.

2. Paradoxalement, le détournement d'une partie des surplus fédéraux vers la caisse d'assurance-vieillesse réduit la marge de manouvre de la classe politique concernant la création de nouveaux programmes sociaux: Glenn Kessler, Exic Pianin, «The Budget's Brick Wall », Washington Post, 7 avril 2000 , p. A1.

3. L'adoption du programme Medicare en 1965 et la centralisation de l'aide sociale destinée aux personnes âgées en 1974 (création du Supplemental Security Income) sont des étapes essentielles de cette centralisation relative, amorcée dès 1935 par l'instauration d'un régime d'assurance-vieillesse entièrement fédéral. 


\section{Béland, F. Vergniolle de Chantal}

tués par l'État fédéral représenterait, par exemple, une concentration excessive de la puissance économique '. L'idée de détourner une partie des surplus fédéraux vers le fonds de réserve du régime d'assurance-vieillesse semble toutefois très populaire auprès de la population, qui se montre généralement très attachée à la préservation de ce régime 2. Dans ce cadre, l'attitude des républicains face à sa « privatisation »même partielle - demeure prudente. En raison de l'importance des enjeux économiques et sociaux liés à la réforme des retraites, les risques électoraux auxquels ils s'exposent paraissent en effet très importants. Accusés à maintes reprises par les démocrates de vouloir « détruire la sécurité sociale», les républicains paraissent craindre de prendre l'initiative sur le plan législatif ${ }^{3}$. Leur attentisme représente une stratégie d'évitement classique. Pour prévenir une réaction négative de l'électorat, il semble toujours préférable de ne rien faire avant la tenue d'importantes élections ${ }^{4}$. Avec les élections présidentielles de novembre 2000, les membres du Congrès n'ont pas dérogé à la règle, et se sont montrés encore plus prudents qu'à 1'habitude ${ }^{5}$. Malgré la virulence du débat politique, la question des retraites se trouve marquée par un immobilisme législatif certain, résultant des stratégies d'évitement républicaines ${ }^{6}$.

La politique de réforme de la protection sociale qui s'est mise en place depuis 1992, résulte en partie de la volonté de Clinton de s'approprier certains thèmes conservateurs. Ses huit ans de présidence ont constitué une tentative pour faire cohabiter cette tactique électorale avec les principes traditionnels du Parti démocrate. Son pragmatisme affiché en 1992 a été exacerbé par le choc des élections de mi-mandat en 1994. Son acceptation de la réforme de 1996 apparaît comme un résultat direct de sa vulnérabilité institutionnelle face à la double majorité républicaine au Congrès. En ce sens, les expériences de réformes de la protection sociale éclairent la nature foncièrement pragmatique des « Nouveaux Démocrates » clintoniens qui, loin de toute cohé-

1. Par exemple, Darcy Ann Olsen, « Social Security Reform Proposals : USAs, Clawbacks, and Other Add-Ons », Washington, Cato Institute, Briefing Papers $n^{\circ} 47,11$ juin 1999.

2. Dans sa thèse, Charles $M$. Brain montre, par exemple, l'attachement durable des classes moyennes envers ce programme : Social Security at the Crossroads: Public Opinion and Public Policy, New York, Garland Publishing, 1991. Tout au long des années 1990, les Américains appuient ce régime tout en formulant des craintes aiguës quant à son avenir budgétaire : Virginia P. Reno, Robert B. Friedland, «Strong Support but Low Confidence : What Explains the Contradiction ? », dans Eric R. Kingson, James H. Schütz (dir.), Social Security in the 21st Century, Oxford, Oxford University Press, 1997, p. 178-195 et Dallas L. Salisbury, « Public Attitudes on Social Security : The UFO Fallacy », EBRI Notes, mars 1998.

3. Par exemple : Richard W. Stevenson, «Lawmakers in Both Parties Are Weighing Compromise Plans to Revamp Social Security », New York Times, 14 janvier 1999, p. A16. Sans l'accord des démocrates, la « privatisation» du régime fédéral semble d'ailleurs improbable. En procédant de façon unilatérale, la majorité républicaine au Congrès s'exposerait aux critiques potentiellement funestes - sur le plan électoral - du Parti démocrate.

4. K. Weaver, «The Politics of Blame Avoidance », art. cité.

5 . Dans le cadre de l'accord budgétaire d'avril 2000 , le Congrès à majorité républicaine accepte finalement de détourner une partie substantielle des surplus budgétaires fédéraux vers la caisse d'assurance-vieillesse : Glenn Kessler, Eric Pianin, «The Budget's Brickwall», Washington Post, 7 avril 2000, p. A1.

6 . Alors que le programme Medicare fait l'objet de réformes périodiques dans le cadre des accords budgétaires entre la Présidence et le Congrès, le régime fédéral d'assurance-vieillesse n'a pas été réformé depuis 1983. 
rence idéologique, se sont, au contraire, adaptés à la conjoncture électorale. Au-delà de ce panorama de la tactique électorale de Clinton et des politiques sociales menées sous sa présidence, la conclusion qui s'impose porte à la fois sur les modèles d'analyse de la réforme des États providence et sur le statut du fédéralisme dans le débat politique américain.

Sur le plan théorique, cette évolution permet de relativiser les analyses, désormais classiques, au sujet de la «nouvelle politique de l'État providence ». Loin de se caractériser par la domination absolue des réductions budgétaires et des stratégies d'évitement, la réforme des politiques sociales sous la présidence Clinton se caractérise par une imbrication de ces stratégies et de la recherche de crédits électoraux. Actuellement, en raison de l'apparition des surplus budgétaires fédéraux, il est de plus en plus clair que les stratégies d'évitement ne résument pas l'ensemble des comportements des acteurs politiques nationaux. Durant la récente campagne présidentielle, les promesses électorales des deux principaux candidats ont attesté de cette recrudescence de la recherche de crédits électoraux dans le champ des politiques sociales, phénomène déjà perceptible avec les discussions entourant le projet clintonien de réforme des retraites '. Mais la portée de ce changement de perception est encore imprécise. L'orientation du discours autour des politiques sociales est toujours marquée par l'élaboration de stratégies d'évitement.

Le fédéralisme se trouve souvent instrumentalisé pour transférer la responsabilité des réductions budgétaires sur les autres niveaux de gouvernement, même si ce type d'emploi n'est pas toujours aussi systématique. La complexité du système fédéral permet bien d'opacifier la responsabilité des acteurs par le jeu des obligations fédérales et de la fragmentation politique induite par l'existence de 50 États fédérés. Dans ce cadre, l'incitation est forte, du point de vue des responsables nationaux, pour transférer le poids des décisions porteuses de risques électoraux sur les niveaux inférieurs de gouvernement. Les réformes sociales discutées et menées sous la présidence Clinton, tout particulièrement la loi sur l'aide sociale de 1996, sont, de ce point de vue, particulièrement révélatrices de cette caractéristique du système fédéral américain. Comme l'a montré R. Kent Weaver, toute démocratie est, dans sa gestion quotidienne, dominée par les stratégies d'évitement ${ }^{2}$. Les hommes politiques de toutes tendances s'efforcent de ne pas endosser la responsabilité de décisions impopulaires. Mais d'autres éléments sont à prendre en considération. Comme nous l'avons vu, la combinaison de la tactique électorale clintonienne et des possibilités offertes par un système fédéral sont de puissantes incitations à la démultiplication de l'emploi des stratégies d'évitement. Au total, l'exemple des réformes sociales sous Clinton nous permet de porter un triple diagnostic sur le débat public américain : non seulement en reconstituant partiellement la nature et la tactique électorale des « Nouveaux Démocrates », mais aussi, plus profondément, en mettant en lumière la logique des réformes sociales et les ambivalences du système fédéral qu'elles révèlent.

En décembre 1999, Daniel Béland a soutenu une thèse de sociologie politique consacrée à la politique des retraites aux États-Unis (EHESS). Il est professeur-adjoint

1. Par exemple, cf. Dan Balz, Terry M. Neal, «Bush Debuts Tax-Cut-and-Spend Strategy », Washington Post, 13 avril 2000. Cf. aussi, Laura Meckler, « Gore Targets Bush on Health Care », Washington Post, 27 avril 2000.

2. K. Weaver, «The Politics of Blame Avoidance », art. cité, p. 390-393. 


\section{Béland, F. Vergniolle de Chantal}

au Département de sociologie et d'anthropologie de l'université Concordia (Montréal, Canada). Parmi ses publications récentes, citons « Expertise et politique des retraites : l'influence des think tanks aux États-Unis », L'année de la régulation, 4, 2000, p. 251274, ainsi que «From Thatcher (and Pinochet) to Clinton ? Conservative Think Tanks, Foreign Models and US Pensions Reform » (en collaboration avec Alex Waddan), Political Quarterly, 71 (2), avril-juin 2000, p. 202-210. Ses travaux actuels portent sur l'histoire et les transformations contemporaines de la protection sociale en Europe ainsi qu'en Amérique du Nord (Département de sociologie et d'anthropologie, Université Concordia, 7141 rue Sherbrooke Ouest, VE-223-2, Montréal, Québec, Canada H4B 1R6 <beland@alcor.concordia.ca>).

François Vergniolle de Chantal est doctorant en science politique à l'Institut d'études politiques de Paris. Il est actuellement ATER (Attaché temporaire d'Enseignement et de Recherche) en civilisation américaine à l'université d'Orléans. Ses publications récentes sont « $L$ 'antifédéralisme américain. Diversité et pérennité », Commentaire, 91 , automne 2000 , p. 543-548; ainsi que « L'analyse constitutionnelle de l'Impeachment aux États-Unis », Revue française de science politique, 50 (1), février 2000, p. 147-154. Il a participé au dernier congrès de l'APSA (American Political Science Association) à Washington DC (31 août-3 septembre 2000) en présentant «Courting Devolution. The Rehnquist Court and Contemporary American Federalism » avec Timothy Conlan, professeur de science politique à l'université George Mason (Virginie, États-Unis). Ses recherches portent sur le caractère antigouvernemental du conservatisme américain depuis les années 1980 (Université d'Orléans, Faculté des Lettres, Langues et Sciences Humaines, Rue de Tours, 45072 Orléans Cedex 2 <cpo@caramail.com $>$ )

\section{RÉSUMÉ/ABSTRACT}

POLITIQUES SOCIALES, STRATÉGIES ÉLECTORALES ET FÉDÉRALISME SOUS LA PRÉSIDENCE CLINTON

L'article se présente comme une analyse des réformes sociales menées sous la présidence Clinton (1993-2001), en se concentrant sur le projet de couverture santé universelle, la réforme de l'aide sociale (1996), et les débats sur le devenir du système dés retraites. Cette étude permet d'avancer un certain nombre de conclusions, tant au plan épistémologique qu'au plan politique. Dans le premier cas, il est possible de relativiser le diagnostic fait par Paul Pierson sur "la nouvelle politique de l'État providence ». Bien loin de se limiter à des politiques d'évitement, les réformes sociales des années 1990 aux États-Unis, comportent une part substantielle de recherche de crédits électoraux. En second lieu, l'exemple des politiques sociales éclaire la nature de l'emploi du thème fédéral dans le débat public. La généralisation de l'évocation du fédéralisme est, dans le cadre de certaines limites, instrumentale et pragmatique, tout comme, d'ailleurs, le thème de la "Troisième Voie » clintonienne.

This article presents an analysis of social policies during the Clinton presidency (1993-2001). Its focus is on health care reform, the Welfare Act of 1996 and the debates about the future of Social Security. It presents various conclusions, both epistemological and political. The developments of social policy during the 1990s in the United States make it possible to downplay Paul Pierson's diagnosis concerning "The New Politics of the Welfare State ». Blame avoi- 


\section{Politiques sociales et stratégies électorales}

dance strategies cannot explain the social policies of the 1990 ; electoral credit-claiming was also an important aspect. Social policies highlight the nature of federalism as an issue in public debates. The generalization of its use is, within certain limits, pragmatic and instrumental. The same can be said for Clinton's "Third Way». 
